\title{
Harmful practices in the management of childhood diarrhea in low- and middle-income countries: a systematic review
}

Emily Carter ${ }^{1 *}$, Jennifer Bryce ${ }^{1}$, Jamie Perin ${ }^{1}$ and Holly Newby ${ }^{2}$

\begin{abstract}
Background: Harmful practices in the management of childhood diarrhea are associated with negative health outcomes, and conflict with WHO treatment guidelines. These practices include restriction of fluids, breast milk and/ or food intake during diarrhea episodes, and incorrect use of modern medicines. We conducted a systematic review of English-language literature published since 1990 to assess the documented prevalence of these four harmful practices, and beliefs, motivations, and contextual factors associated with harmful practices in low- and middle-income countries.

Methods: We electronically searched PubMed, Embase, Ovid Global Health, and the WHO Global Health Library. Publications reporting the prevalence or substantive findings on beliefs, motivations, or context related to at least one of the four harmful practices were included, regardless of study design or representativeness of the sample population.
\end{abstract}

Results: Of the 114 articles included in the review, 79 reported the prevalence of at least one harmful practice and 35 studies reported on beliefs, motivations, or context for harmful practices. Most studies relied on sub-national population samples and many were limited to small sample sizes. Study design, study population, and definition of harmful practices varied across studies. Reported prevalence of harmful practices varied greatly across study populations, and we were unable to identify clearly defined patterns across regions, countries, or time periods. Caregivers reported that diarrhea management practices were based on the advice of others (health workers, relatives, community members), as well as their own observations or understanding of the efficacy of certain treatments for diarrhea. Others reported following traditionally held beliefs on the causes and cures for specific diarrheal diseases.

Conclusions: Available evidence suggests that harmful practices in diarrhea treatment are common in some countries with a high burden of diarrhea-related mortality. These practices can reduce correct management of diarrheal disease in children and result in treatment failure, sustained nutritional deficits, and increased diarrhea mortality. The lack of consistency in sampling, measurement, and reporting identified in this literature review highlights the need to document harmful practices using standard methods of measurement and reporting for the continued reduction of diarrhea mortality.

\footnotetext{
* Correspondence: ecarter@jhu.edu

${ }^{1}$ Institute for International Programs, Johns Hopkins Bloomberg School of

Public Health, 615 North Wolfe Street, Baltimore, MD 21205, USA

Full list of author information is available at the end of the article
} 


\section{Background}

Diarrheal disease is a leading cause of mortality in children under five, resulting in around 750,000 deaths each year [1]. The WHO recommends first line management of diarrhea in children under five with continued feeding, increased fluids, and supplemental zinc for 10-14 days to prevent dehydration. In addition, the WHO guidelines state that children exhibiting non-severe dehydration should "receive oral rehydration therapy (ORT) with ORS solution in a health facility". Antimicrobials are recommended only for the treatment of bloody diarrhea or suspected cholera with severe dehydration [2]. The full guidelines, which have evolved over time, are available at http://www.who.int/entity/maternal_child_adolescent/ documents/9241593180/en/index.html.

For decades, health initiatives have targeted the expansion of ORS and ORT, including the UNICEF Growth Monitoring, Oral Rehydration, Breastfeeding and Immunization (GOBI) initiative, the USAID/CDC Africa Child Survival Initiative - Combatting Childhood Communicable Diseases (ACSI-CCCD), and the WHO Integrated Management of Childhood Illness (IMCI) initiative. Despite these efforts, a shift in global attention away from diarrhea management seems likely to have contributed to slowing - and even reversals - in progress toward full coverage for ORT $[3,4]$.

Many fewer programs have specifically targeted nonadherence to other recommended diarrhea management practices, such as the restriction of fluids, breast milk and/ or food intake during diarrhea episodes, and incorrect use of modern medicines. All four of these practices are associated with negative outcomes and conflict with WHO treatment guidelines. Curtailment of fluids and restriction of feeding during diarrhea can increase the risk of dehydration, reduce nutritional intake, and potentially inhibit child growth and development. The use of antibiotics and other medications is appropriate only in the treatment of cholera or dysenteric diarrhea in children. Antidiarrheal drugs and some antiemetics not only have no benefit in diarrhea treatment, but may also cause serious, even lifethreatening side effects in children [2]. We have referred to these as "harmful practices" from this point forward, understanding that under some circumstances these practices may not be detrimental.

This review summarizes existing literature on harmful practices in diarrhea case management in children under five years of age, including fluid and breastfeeding curtailment, food restriction, and inappropriate use of medications for diarrhea management in children in low- and middle-income countries. The primary objectives of the review are to:

- Determine the documented prevalence of these four harmful practices across low- and middle-income populations, as reported in various studies since 1990;

- Describe how these practices have been examined and reported on previously;

- Explore beliefs, motivations, and contextual factors associated with harmful practices as reported through both quantitative and qualitative studies; and

- Highlight associations between these harmful practices and other characteristics of the episode, child, caregiver, and household.

Findings from this review will identify critical next steps to address harmful practices in diarrhea management and ultimately improve child survival.

\section{Methods}

We searched PubMed, Embase, Ovid Global Health, and the WHO Global Health Library in September 2013. Papers were identified that included variations on the combination of the following terms within the publication's title or abstract or as a keyword: 1) diarrhea; 2) low- and middle-income country; and one or more terms related to 3) a harmful practice or general management of diarrhea. Search terms were developed in PubMed (see Additional file 1) and translated for the three other databases. Publications were restricted to English-language articles published after 1990.

Quantitative articles were included if the paper reported the prevalence of at least one of the four harmful practices associated with caregiver management of diarrhea in children under the age of five, regardless of study design or representativeness of the sample population. Qualitative articles, or quantitative articles not meeting the quantitative inclusion criteria, were included if they presented substantive findings on beliefs, motivations, or context related to at least one of the four practices in caregiver management of childhood diarrhea. Publications were excluded if they exclusively reported data collected prior to 1990, exclusively reported provider practices, reported findings post-intervention only, or did not specifically focus on treatment of children under 5 years of age. Due to the variety of study designs included in the review, study quality was not formally assessed, because multiple quality assessment frameworks would have been required.

Data extraction was completed by the first author (EC). For all studies, information on the study design, study population, and sample size was extracted. For studies reporting prevalence of practices, data were extracted on the definition of the practice measure, the reported prevalence of the practice, and variation in the practice by other factors (reported as stratified prevalence or odds ratio). For non-prevalence studies, data were extracted related to 
beliefs, motivations, or context directly related to one or more of the harmful practices and then classified by common themes.

We summarize the results for each of the four harmful practices in the results section of the manuscript. For each practice, we: (1) describe how the practice was defined and measured in these studies; (2) summarize reported findings on prevalence, including variations by characteristics of the diarrhea episode, child, caregiver, and household; and (3) report on beliefs, motivations, and contextual factors investigated and relevant results.

\section{Results}

The initial search yielded 2,266 articles in Pubmed, 2,512 articles in Embase, 1,512 articles in Ovid Global Health, and 1,890 articles in the WHO Global Health Library. After removing duplicates, 4,270 unique articles remained. Title and abstract review and full article review were conducted by the first author (EC). After reviewing titles and abstracts, 294 articles were identified for full article review. Based on a review of the full article, 157 articles did not meet the inclusion criteria and a full text copy of 23 manuscripts could not be located. In total, 114 publications met the inclusion criteria and were included in the review (Fig. 1). Of the 79 studies reporting the prevalence of at least one harmful practice, 54 studies utilized a population-based cross-sectional sample (3 nationally representative), 12 studies used a non-cross-sectional design but included a representative population sample, and 13 studies employed a nonrepresentative sample. Of the 35 studies reporting on beliefs, motivations, or context for harmful practices, 9 studies used exclusively qualitative methods, 8 studies used mixed-methods, and 18 studies used exclusively quantitative methods (12 with a representative sample, 6 with a non-representative sample). Although there have been summaries of relevant Demographic and Health Survey (DHS) and Multiple Indicator Cluster Survey

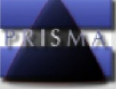

PRISMA 2009 Flow Diagram
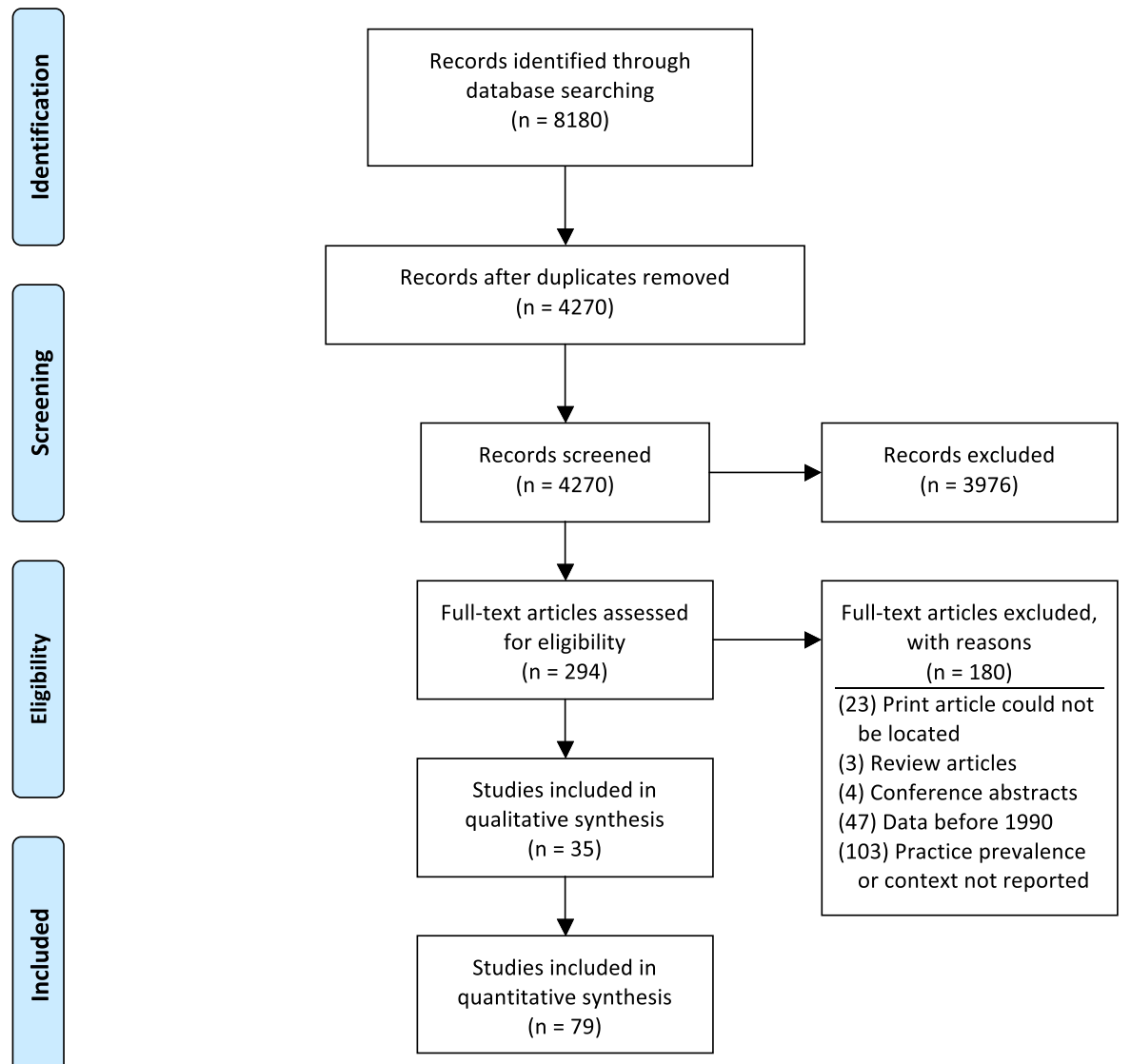

Fig. 1 Flow of studies considered in the systematic review 
(MICS) findings [5, 6], we were unable to identify any country-specific secondary analyses on this topic.

\section{Study characteristics}

The publication dates of the 114 studies included in the review were relatively evenly distributed over the period from 1990 to 2013, with publications clustering slightly in the early 1990s and late 2000s/early 2010s. The majority of studies were conducted in South Asia and subSaharan Africa (Fig. 2). The number of publications reporting on the prevalence of each of the four practices varied, with the highest proportion reporting on inappropriate medication use (70\%), followed in order of frequency by food restriction (56\%), curtailment of fluids other than breast milk (53\%), and breastfeeding restriction (37\%).

Respondents in the majority of prevalence studies were caregivers of children under 5 years of age, although some studies interviewed mothers exclusively. The age of children referenced for the practice also varied, with the majority of studies referencing children under 5 years of age. The definition of the diarrhea reference episode also varied, ranging from diarrhea in the past $24 \mathrm{~h}$ to the most recent diarrhea event, although the most common reference period was the previous two weeks.

\section{Fluid curtailment}

The measurement of fluid intake, and prevalence estimates, varied widely across studies (Table 1 , Column 4 ). Many studies differed in their definition or failed to specify if fluid restriction included or excluded breastfeeding or assessed amount of fluid offered versus consumed. The reported practice of curtailing fluids during a recent episode of diarrhea ranged from as low as $11 \%$ of caregivers in Mirzapur, Bangladesh [7] to over $80 \%$ of caregivers in Kenya's Nyanza province [8]. Where specified by the study authors, the practice of stopping all fluids was uncommon, generally reported in fewer than $10 \%$ of episodes.

Multiple studies explored variations in fluid curtailment by characteristics of the diarrhea episode, child, caregiver, and household (Table 2). Fluid curtailment was associated with diarrhea severity and vomiting in two studies $[9,10]$, whereas increase in fluid was associated with long illness duration and poor appetite [11]. Studies in Pakistan, Bangladesh, and Saudi Arabia found no clear association between fluid restriction and the age of the child [12-14]. However, a study in Mozambique reported that less fluid was given to infants relative to older children [15]. Younger mothers and mothers who did not work outside the home [12] and less educated mothers [16] were more likely to curtail fluids.

Multiple studies have attributed the practice of fluid curtailment to caregiver beliefs about the impact of fluid intake on a child's diarrhea episode (Table 3). Multiple studies reported that caregivers often stated that more or specific fluids would increase the severity of the illness [17-19] or could not be digested [20-22]. Two studies suggested these beliefs were informed by caregivers' observations that reduced fluids decreased stool output and diarrhea intensity $[7,23]$. One study reported that certain types of diarrhea are perceived to be manageable by adjusting fluid intake, while others require traditional or spiritual methods, or no treatment at all

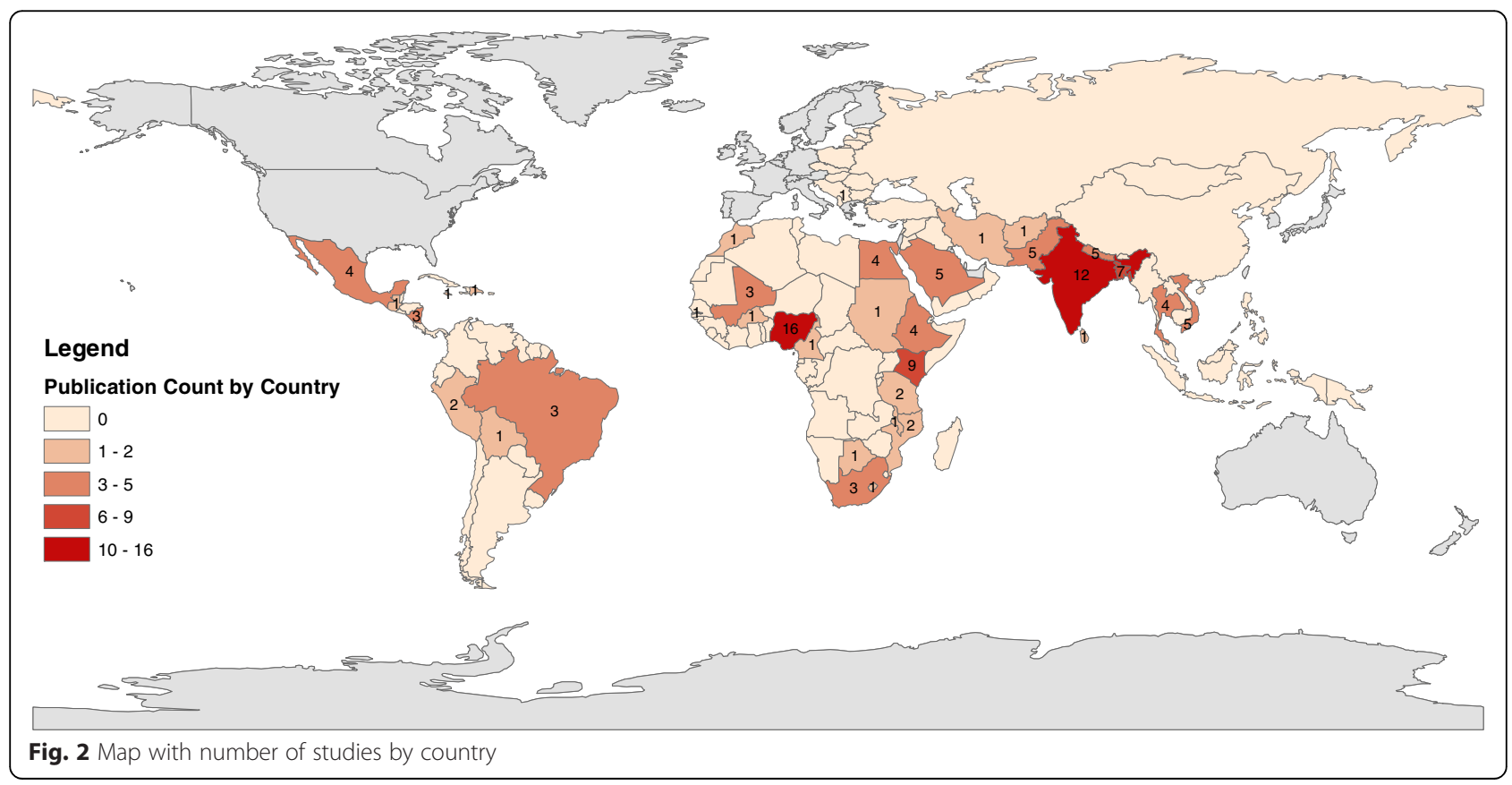


Table 1 Prevalence of harmful practices by region and country

\begin{tabular}{|c|c|c|c|c|c|c|c|c|c|c|}
\hline $\begin{array}{l}\text { Author, } \\
\text { Year [reference] }\end{array}$ & Country & $\begin{array}{l}\text { Study design, study population, } \\
\text { number of participants }\end{array}$ & $\begin{array}{l}\text { Proportion restricting } \\
\text { fluid }\end{array}$ & & $\begin{array}{l}\text { Proportion restricting } \\
\text { breastfeeding }\end{array}$ & & $\begin{array}{l}\text { Proportion restricting } \\
\text { food }\end{array}$ & & Proportion using drugs & \\
\hline \multicolumn{11}{|l|}{ Americas } \\
\hline $\begin{array}{l}\text { Emond et al., } \\
2002 \text { [84] }\end{array}$ & Brazil & $\begin{array}{l}\text { Cross-sectional baseline survey } \\
\text { preceding intervention, Northeast } \\
\text { Brazil 1997, Caregivers of children } \\
\text { with diarrhea in the previous } \\
2 \text { days, } n=922\end{array}$ & & & & & & & $\begin{array}{l}\text { Generally give } \\
\text { medicines other } \\
\text { than ORS }\end{array}$ & 7 \\
\hline \multirow[t]{2}{*}{$\begin{array}{l}\text { Strina et al., } \\
2005[63]\end{array}$} & \multirow[t]{2}{*}{ Brazil } & \multirow{2}{*}{$\begin{array}{l}\text { Longitudinal survey, Salvador } \\
\text { 1997-1999, Caregivers of } \\
\text { children } \leq 36 \text { months with } \\
\text { diarrhea in previous } 2 \text { weeks, } \\
n=2403 \text { episodes }\end{array}$} & & & & & & & $\begin{array}{l}\text { Gave industrial } \\
\text { medicines }\end{array}$ & 40.9 \\
\hline & & & & & & & & & $\begin{array}{l}\text { Gave industrial } \\
\text { medicines \& home } \\
\text { preparation }\end{array}$ & 2.7 \\
\hline $\begin{array}{l}\text { Webb et al., } \\
2010 \text { [85] }\end{array}$ & Guatemala & $\begin{array}{l}\text { Longitudinal survey, Population } \\
\text { of Spanish-Mayan Descent } \\
\text { 1996-1999, Caregivers of children } \\
<36 \text { months with diarrhea in } \\
\text { previous } 19 \text { days, } n=466\end{array}$ & Stopped or less fluid ${ }^{a}$ & 55 & $\begin{array}{l}\text { Stopped or less } \\
\text { breastfeeding }^{\mathrm{b}}\end{array}$ & 26.6 & $\begin{array}{l}\text { Stopped or less } \\
\text { food }\end{array}$ & 15 & & \\
\hline $\begin{array}{l}\text { Bachrach et al., } \\
2002 \text { [21] }\end{array}$ & Jamaica & $\begin{array}{l}\text { Case-control hospital based survey, } \\
\text { Kingston } 2007 \text {, Caregivers of } \\
\text { children }<5 \text { years presenting at } \\
\text { hospital, } n=215 \text { total, } 117 \\
\text { gastroenteritis cases }\end{array}$ & & & & & & & $\begin{array}{l}\text { Child presenting } \\
\text { with gastroenteritis: } \\
\text { Gave antidiarrheal/ } \\
\text { antimotility drug } \\
\text { before coming to } \\
\text { hospital }\end{array}$ & 36 \\
\hline \multirow[t]{2}{*}{$\begin{array}{l}\text { Martinez et al., } \\
1991 \text { [52] }\end{array}$} & \multirow[t]{2}{*}{ Mexico } & \multirow{2}{*}{$\begin{array}{l}\text { Cross-sectional survey, Rural } \\
\text { Highlands of Central Mexico } \\
\text { (year not specified), Caregivers } \\
\text { of children }<5 \text { years, diarrhea } \\
\text { episode reference unclear, } n=38\end{array}$} & & & & & & & $\begin{array}{l}\text { Give pill as first } \\
\text { treatment for diarrhea }\end{array}$ & 47 \\
\hline & & & & & & & & & $\begin{array}{l}\text { Give over-the-counter } \\
\text { drug to child }\end{array}$ & 53 \\
\hline \multirow[t]{2}{*}{$\begin{array}{l}\text { Perez-Cuevas } \\
\text { et al., } 1996 \text { [40] }\end{array}$} & \multirow[t]{2}{*}{ Mexico } & \multirow{2}{*}{$\begin{array}{l}\text { Cross-sectional survey, Tiaxcala } \\
\text { (year not specified), Caregivers } \\
\text { of children }<5 \text { years with diarrhea } \\
\text { in previous } 2 \text { weeks, } n=747\end{array}$} & $\begin{array}{l}\text { "Withheld" non-breast } \\
\text { milk }\end{array}$ & 27.2 & $\begin{array}{l}\text { Stopped } \\
\text { breastfeeding }^{b}\end{array}$ & 12.2 & $\begin{array}{l}\text { Stopped or reduced } \\
\text { food other than milk }\end{array}$ & 9.1 & Treated with any drug & 35.2 \\
\hline & & & No liquids given & 3 & & & $\begin{array}{l}\text { Any dietary } \\
\text { restriction }\end{array}$ & 36.6 & & \\
\hline \multirow{3}{*}{$\begin{array}{l}\text { Martinez et al., } \\
1998 \text { [86] }\end{array}$} & \multirow[t]{3}{*}{ Mexico } & \multirow{3}{*}{$\begin{array}{l}\text { Cross-section of ethnographic } \\
\text { study participants, } 3 \text { States } \\
\text { (year not specified), Caregivers } \\
\text { of children }<5 \text { years in reference } \\
\text { to most recent diarrhea episode, } \\
n=186\end{array}$} & & & & & & & Gave antimicrobial & 37.1 \\
\hline & & & & & & & & & Gave antidiarrheal & 28 \\
\hline & & & & & & & & & Gave antipyretic & 18 \\
\hline $\begin{array}{l}\text { Smith et al., } \\
1993 \text { [51] }\end{array}$ & Nicaragua & $\begin{array}{l}\text { Cross-sectional survey, Rural Pacific } \\
\text { Coastal Plain (year not specified), } \\
\text { Caregivers of infants, diarrhea } \\
\text { episode reference unclear, } n=70\end{array}$ & & & $\begin{array}{l}\text { Stopped breastfeeding } \\
\text { (among those who }^{\text {reported changing }} \\
\text { feeding) }^{b}\end{array}$ & 4 & $\begin{array}{l}\text { Did not give solid } \\
\text { foods (among those } \\
\text { who reported } \\
\text { changing feeding) }\end{array}$ & 13 & & \\
\hline
\end{tabular}


Table 1 Prevalence of harmful practices by region and country (Continued)

\begin{tabular}{|c|c|c|c|c|c|c|c|c|c|c|}
\hline \multirow{3}{*}{$\begin{array}{l}\text { Gorter et al., } \\
1995 \text { [79] }\end{array}$} & \multirow[t]{3}{*}{ Nicaragua } & \multirow{3}{*}{\multicolumn{7}{|c|}{$\begin{array}{l}\text { Cross-section of ethnographic study } \\
\text { participants, Rural Pacific Coastal } \\
\text { Plain 1990, Caregivers of children } \\
<5 \text { years with diarrhea in previous } \\
2 \text { weeks, } n=216\end{array}$}} & Gave antibiotic & 22 \\
\hline & & & & & & & & & $\begin{array}{l}\text { Gave parasite } \\
\text { medicine }\end{array}$ & 19 \\
\hline & & & & & & & & & Gave laxative & 6 \\
\hline $\begin{array}{l}\text { Vazquez et al., } \\
2002 \text { [33] }\end{array}$ & Nicaragua & $\begin{array}{l}\text { Cross-sectional survey, North of } \\
\text { Central Region 1990, Caregivers of } \\
\text { children }<5 \text { years with diarrhea in } \\
\text { previous } 2 \text { weeks, } n=187\end{array}$ & & & & & $\begin{array}{l}\text { Child ate less than } \\
\text { usual }\end{array}$ & 43.5 & $\begin{array}{l}\text { Gave any } \\
\text { pharmaceutical }\end{array}$ & 60 \\
\hline $\begin{array}{l}\text { Kristiansson et al., } \\
2009 \text { [87] }\end{array}$ & Peru & $\begin{array}{l}\text { Cross-sectional survey, Yurimaguas } \\
\text { and Moyobamba Departments 2002, } \\
\text { Caregivers of children } 6-72 \text { months } \\
\text { with illness in previous } 2 \text { weeks, } \\
n=780\end{array}$ & & & & & & & $\begin{array}{l}\text { Antibiotic use } \\
\text { reported by wealth } \\
\text { quintile only }\end{array}$ & \\
\hline \multicolumn{11}{|l|}{ Europe } \\
\hline \multirow[t]{2}{*}{$\begin{array}{l}\text { Berisha et al., } \\
2009 \text { [16] }\end{array}$} & \multirow[t]{2}{*}{ Kosovo } & \multirow{2}{*}{$\begin{array}{l}\text { Cross-sectional survey, Kosovo 2005, } \\
\text { Mothers of children }<5 \text { years in } \\
\text { reference to most recent diarrhea } \\
\text { episode, } n=107\end{array}$} & Less fluid or none & 62.6 & $\begin{array}{l}\text { Stopped or reduced } \\
\text { amount of food or } \\
\text { breastfeeding }\end{array}$ & 43.9 & & & & \\
\hline & & & Same fluids ${ }^{a}$ & 19.6 & $\begin{array}{l}\text { Same amount of food } \\
\text { or breastfeeding }\end{array}$ & 48.6 & & & & \\
\hline \multicolumn{11}{|l|}{$\begin{array}{l}\text { Eastern } \\
\text { Mediterranean }\end{array}$} \\
\hline $\begin{array}{l}\text { Azim et al., } \\
1993 \text { [37] }\end{array}$ & Afghanistan & $\begin{array}{l}\text { Cross-sectional study, Paktika } \\
\text { Province 1991, Caregivers of } \\
\text { children }<5 \text { years with diarrhea in } \\
\text { previous } 2 \text { weeks, } n=87\end{array}$ & Same or less fluid ${ }^{d}$ & 43.7 & $\begin{array}{l}\text { Stopped } \\
\text { breastfeeding }\end{array}$ & 5.9 & Stopped or less food & 33.5 & Gave any drug & 66 \\
\hline \multirow[t]{4}{*}{$\begin{array}{l}\text { Langsten et al., } \\
1994 \text { [88] }\end{array}$} & \multirow[t]{4}{*}{ Egypt } & \multirow{4}{*}{$\begin{array}{l}\text { Longitudinal survey, Lower Egypt } \\
\text { 1990, Caregivers of children }<5 \text { years } \\
\text { with diarrhea in previous } 2 \text { weeks, } \\
n=4900\end{array}$} & $\begin{array}{l}\text { Stopped fluids other } \\
\text { than BF and } \text { milk }^{d}\end{array}$ & 2.8 & $\begin{array}{l}\text { Stopped } \\
\text { breastfeeding }\end{array}$ & 2.5 & Stopped food & 5.8 & & \\
\hline & & & Reduced other fluids ${ }^{d}$ & 10.9 & $\begin{array}{l}\text { Decreased } \\
\text { breastfeeding }\end{array}$ & 11.9 & Reduced food & 22.7 & & \\
\hline & & & $\begin{array}{l}\text { Reduced non-breast } \\
\text { milk }^{d}\end{array}$ & 15.3 & & & & & & \\
\hline & & & $\begin{array}{l}\text { Stopped non-breast } \\
\text { milk }^{\mathrm{d}}\end{array}$ & 9.9 & & & & & & \\
\hline
\end{tabular}


Table 1 Prevalence of harmful practices by region and country (Continued)

\begin{tabular}{|c|c|c|c|c|c|c|c|c|c|c|}
\hline \multirow[t]{8}{*}{$\begin{array}{l}\text { Langsten et al., } \\
1995 \text { [57] }\end{array}$} & \multirow[t]{8}{*}{ Egypt } & \multirow{8}{*}{$\begin{array}{l}\text { Longitudinal survey, Lower Egypt } \\
\text { 1990-1991, Caregivers of children } \\
<5 \text { years with diarrhea in previous } \\
2 \text { weeks, } n=4900\end{array}$} & & & & & & & $\begin{array}{l}\text { Among acute } \\
\text { non-dysenteric cases: } \\
\text { Used antibiotics }\end{array}$ & 46.5 \\
\hline & & & & & & & & & $\begin{array}{l}\text { Among acute } \\
\text { non-dysenteric cases: } \\
\text { Used antibiotics only }\end{array}$ & 3.2 \\
\hline & & & & & & & & & $\begin{array}{l}\text { Among acute } \\
\text { non-dysenteric cases: } \\
\text { Used other medicine }\end{array}$ & 63.3 \\
\hline & & & & & & & & & $\begin{array}{l}\text { Among acute } \\
\text { non-dysenteric cases: } \\
\text { Used other medicine } \\
\text { only }\end{array}$ & 18.6 \\
\hline & & & & & & & & & $\begin{array}{l}\text { Among all cases: } \\
\text { Used antibiotics }\end{array}$ & 45.6 \\
\hline & & & & & & & & & $\begin{array}{l}\text { Among all cases: } \\
\text { Used antibiotics only }\end{array}$ & 3.4 \\
\hline & & & & & & & & & $\begin{array}{l}\text { Among all cases: } \\
\text { Used other medicine }\end{array}$ & 63.0 \\
\hline & & & & & & & & & $\begin{array}{l}\text { Among all cases: } \\
\text { Used other medicine } \\
\text { only }\end{array}$ & 19.3 \\
\hline \multirow[t]{3}{*}{$\begin{array}{l}\text { Jousilahti et al., } \\
1992 \text { [75] }\end{array}$} & \multirow[t]{3}{*}{ Egypt } & \multirow{3}{*}{$\begin{array}{l}\text { Cross-sectional cluster study, Lower } \\
\text { Egypt 1992, Caregivers of children } \\
<5 \text { years with diarrhea in previous } \\
24 \mathrm{~h}, \mathrm{n}=766\end{array}$} & Same or less fluid ${ }^{d}$ & 75.6 & $\begin{array}{l}\text { Stopped } \\
\text { breastfeeding }^{\mathrm{b}}\end{array}$ & 3.7 & $\begin{array}{l}\text { Stopped or less } \\
\text { solid or semi-solid } \\
\text { food }\end{array}$ & 30.2 & Gave any drug & 54.2 \\
\hline & & & & & & & & & Gave drug and ORS & 17.6 \\
\hline & & & & & & & & & $\begin{array}{l}\text { Gave drug but } \\
\text { no ORS }\end{array}$ & 36.5 \\
\hline \multirow{8}{*}{$\begin{array}{l}\text { El-Gllany et al., } \\
2005 \text { [62] }\end{array}$} & \multirow[t]{8}{*}{ Egypt } & \multirow{8}{*}{$\begin{array}{l}\text { Cross-sectional study, Dakahalia } \\
2002-2003 \text {, Caregivers of children } \\
<5 \text { years with diarrhea in previous } \\
2 \text { weeks, } n=1052\end{array}$} & Same or less fluide & \multirow[t]{8}{*}{29} & & & Stopped feeding ${ }^{\mathrm{e}}$ & 12.7 & Gave any drug & 74.7 \\
\hline & & & & & & & & & $\begin{array}{l}\text { Among those } \\
\text { receiving a drug: }\end{array}$ & 36.9 \\
\hline & & & & & & & & & Antibiotic $^{f}$ & 73.9 \\
\hline & & & & & & & & & Antidiarrheal $^{f}$ & 73.9 \\
\hline & & & & & & & & & Antiemetic $^{f}$ & 16.7 \\
\hline & & & & & & & & & Antiprotozoal ${ }^{f}$ & 5.7 \\
\hline & & & & & & & & & Antipyretic $^{f}$ & 9.6 \\
\hline & & & & & & & & & Antispasmodic ${ }^{f}$ & 1.7 \\
\hline
\end{tabular}


Table 1 Prevalence of harmful practices by region and country (Continued)

\begin{tabular}{|c|c|c|c|c|c|c|c|c|c|c|}
\hline $\begin{array}{l}\text { Amini-Ranjbar } \\
\text { et al., } 2007 \text { [53] }\end{array}$ & Iran & $\begin{array}{l}\text { Cross-sectional study, Kerman } \\
2005, \text { Caregivers of children } \\
6-24 \text { months with diarrhea in } \\
\text { previous } 2 \text { months, } n=330\end{array}$ & & & $\begin{array}{l}\text { Same or less } \\
\text { breastfeeding }\end{array}$ & 53.8 & $\begin{array}{l}\text { Decreased solid } \\
\text { foods }\end{array}$ & 20 & & \\
\hline WHO, 1991 [89] & Morocco & $\begin{array}{l}\text { Cross-sectional study, National 1990, } \\
\text { Caregivers of children }<5 \text { years } \\
\text { with diarrhea in previous } 24 \text { h, } \\
n=1066\end{array}$ & Same or less fluid & 70 & & & & & Gave any drug & 22.6 \\
\hline \multirow{2}{*}{$\begin{array}{l}\text { Morisky et al., } \\
2002 \text { [90] }\end{array}$} & \multirow[t]{2}{*}{ Pakistan } & \multirow{2}{*}{$\begin{array}{l}\text { Cross-sectional survey, National } \\
\text { 1991-1992, Caregivers of children } \\
<2 \text { years in reference to most } \\
\text { recent episode, } n=5433\end{array}$} & \multirow[t]{2}{*}{ Stop fluids ${ }^{\mathrm{e}}$} & \multirow[t]{2}{*}{9.2} & & & Stopped food & 5.9 & Gave antibiotic & 11 \\
\hline & & & & & & & Reduced food & 6.2 & Gave other medicine & 9.2 \\
\hline $\begin{array}{l}\text { Quadri et al., } \\
2013 \text { [13] }\end{array}$ & Pakistan & $\begin{array}{l}\text { Cross-sectional study (HUAS), } \\
\text { Low-Income peri-urban area near } \\
\text { Karachi 2007, Caregivers of children } \\
<5 \text { years with diarrhea in previous } \\
2 \text { weeks, } n=959\end{array}$ & $\begin{array}{l}\text { Did not offer } \\
\text { "to drink" (at home } \\
\text { before seeking care) }\end{array}$ & 22.5 & & & $\begin{array}{l}\text { Did not offer } \\
\text { "to eat" (at home } \\
\text { before seeking care) }\end{array}$ & 44.1 & $\begin{array}{l}\text { Gave antibiotic } \\
\text { (at home) }\end{array}$ & 7.7 \\
\hline $\begin{array}{l}\text { Nasrin et al., } \\
2013 \text { [91] }\end{array}$ & Pakistan & $\begin{array}{l}\text { Cross-sectional study (HUAS), } \\
\text { Low-Income periurban area near } \\
\text { Karachi 2007, Caregivers of children } \\
<5 \text { years with diarrhea in previous } \\
2 \text { weeks, } n=349\end{array}$ & $\begin{array}{l}\text { Offered same or less } \\
\text { than usual to drink }\end{array}$ & 33.9 & & & $\begin{array}{l}\text { Offered less than } \\
\text { usual to eat }{ }^{\mathrm{e}}\end{array}$ & 33.6 & & \\
\hline $\begin{array}{l}\text { Bella et al., } \\
1994 \text { [92] }\end{array}$ & Saudi Arabia & $\begin{array}{l}\text { Case-control study, Eastern Province } \\
\text { (year not specified), Caregiver of } \\
\text { infant with diarrhea at time of } \\
\text { survey versus caregiver of infant } \\
\text { without diarrhea, } n=344 \text { total, } \\
68 \text { cases }\end{array}$ & $\begin{array}{l}\text { Stopped bottle } \\
\text { feeding (among } \\
\text { cases who were } \\
\text { bottle feeding) }\end{array}$ & 35 & & & & & & \\
\hline \multirow{2}{*}{$\begin{array}{l}\text { al-Mazrou et al., } \\
1995 \text { [93] }\end{array}$} & \multirow[t]{2}{*}{ Saudi Arabia } & \multirow{2}{*}{$\begin{array}{l}\text { Cross-sectional survey, National } \\
1991 \text {, Caregivers of children } \\
<5 \text { years with diarrhea in the } \\
\text { previous } 2 \text { weeks, } n=6300 \\
\text { screened }\end{array}$} & & & & & & & Gave drugs & 40.7 \\
\hline & & & & & & & & & Gave IV fluids & 4.7 \\
\hline \multirow[t]{2}{*}{$\begin{array}{l}\text { Bani et al., } \\
2002 \text { [12] }\end{array}$} & \multirow[t]{2}{*}{ Saudi Arabia } & \multirow{2}{*}{$\begin{array}{l}\text { Cross-sectional hospital based } \\
\text { survey, Riyadh City (year not } \\
\text { specified), Mothers of children } \\
\leq 24 \text { months with diarrhea } \\
\text { attending primary health clinic, } \\
n=237\end{array}$} & Less fluid given ${ }^{\mathrm{e}}$ & 11.3 & $\begin{array}{l}\text { Less frequency of } \\
\text { breastfeeding }\end{array}$ & 24.6 & $\begin{array}{l}\text { Less solid/semi-solid } \\
\text { food given }\end{array}$ & 22.7 & & \\
\hline & & & Same fluid given ${ }^{e}$ & 13.2 & $\begin{array}{l}\text { Same frequency of } \\
\text { breastfeeding }^{\mathrm{b}}\end{array}$ & 37.7 & $\begin{array}{l}\text { Same solid/ } \\
\text { semi-solid food } \\
\text { given }\end{array}$ & 22.6 & & \\
\hline $\begin{array}{l}\text { Moawed et al., } \\
2000 \text { [20] }\end{array}$ & Saudi Arabia & $\begin{array}{l}\text { Cross-sectional hospital based } \\
\text { survey, Riyadh City 1998, Mothers } \\
\text { of infants with diarrhea attending } \\
2 \text { pediatric hospital diarrhea } \\
\text { centers, } n=300\end{array}$ & & & $\begin{array}{l}\text { Stop breastfeeding or } \\
\text { milk feeding }\end{array}$ & 62 & & & $\begin{array}{l}\text { Gave unprescribed } \\
\text { medicine }\end{array}$ & 38 \\
\hline
\end{tabular}


Table 1 Prevalence of harmful practices by region and country (Continued)

\section{Africa}

Wilson et al $2012[11]$

Burkina Faso Cross-sectional survey, Orodara Health District 2012, Primary caregivers of children $<27$ months with diarrhea in previous 2 weeks, $n=1067$

Olango et al., Ethiopia
1990 [17]

Cross-sectional survey, Rural (year not specified), Mothers of children $<5$ years with diarrhea in previous 2 weeks, $n=619$

\begin{tabular}{|c|c|c|}
\hline $\begin{array}{l}\text { Ketsela et al., } \\
1991 \text { [94] }\end{array}$ & Ethiopia & $\begin{array}{l}\text { Cross-sectional survey, Shewa } \\
\text { Administrative Regions } 1990, \\
\text { Mothers of children }<5 \text { years, } \\
\text { diarrhea episode reference } \\
\text { unclear, } n=750\end{array}$ \\
\hline $\begin{array}{l}\text { Mash et al., } \\
2003 \text { [95] }\end{array}$ & Ethiopia & $\begin{array}{l}\text { Cross-sectional survey, Oromia } \\
\text { Region 1997, Caregivers of children } \\
<24 \text { months with diarrhea in the } \\
\text { previous fortnight, } n=111\end{array}$ \\
\hline $\begin{array}{l}\text { Mediratta et al., } \\
2010 \text { [9] }\end{array}$ & Ethiopia & $\begin{array}{l}\text { Case-control hospital based study, } \\
\text { Gondar } 2007 \text {, Caregivers of children } \\
<5 \text { years with diarrhea attending } \\
\text { referral hospital, case } n=220\end{array}$ \\
\hline $\begin{array}{l}\text { Saha et al., } \\
2013 \text { [96] }\end{array}$ & Gambia & $\begin{array}{l}\text { Cross-sectional survey, Upper River } \\
\text { Region } 2009 \text {, Caregivers of children } \\
<5 \text { years with diarrhea in the } \\
\text { previous } 2 \text { weeks, } n=258\end{array}$ \\
\hline
\end{tabular}

Oyoo et al., Kenya 1991 [39] across Kenya 1990, Caregivers of population in Wolayta district
Same or less fluid

(breastfed children

separate category

within fluid intake

measure)

\section{Decreased fluids}

Same amount

of fluids

No fluids ${ }^{\mathrm{a}}$

Less than usual fluid ${ }^{a}$

Same as usual fluid ${ }^{a}$

Stopped or

decreased fluids ${ }^{a}$

Less of other fluids ${ }^{a}$

Same amount

Same or less fluid
Stopped fluids
64.1 Stopped

breastfeeding $^{b}$

42.3

10.3

268 No breastfeeding

31.4

23.8

47.7 Stopped or decreased 67.6 breastfeeding $^{b}$

29 Gave less breast milk 24

44 Same amount of 34 breast milk ${ }^{\mathrm{b}}$

36.1

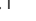

Less than usual amount of food
74 - Stopped
96 breastfeeding

diet $^{\mathrm{e}}$

Stopped food

(not weaned are

Decreased food

Same amount

of food

as usual ${ }^{\mathrm{c}}$

Gave no food ${ }^{c}$

Stopped or less food

"Withheld" food additional category)

54.4

3.5 Gave less fluid than ${ }^{c} \quad 35.9$

Gave same fluid $\quad 38.2$ (at home)

Gave antimicrobia (among those seeking care at health facility)

Gave injectable medicine (among those seeking care at health facility)

Same or less fluid children $<5$ years with diarrhea in the previous 2 weeks, $n=23884$ screened 
Table 1 Prevalence of harmful practices by region and country (Continued)

\begin{tabular}{|c|c|c|c|c|c|c|c|c|c|c|}
\hline $\begin{array}{l}\text { Mirza et al., } \\
1997 \text { [97] }\end{array}$ & Kenya & $\begin{array}{l}\text { Longitudinal study with } 24 \mathrm{~h} \\
\text { dietary recall, Kibera Slum } \\
\text { 1989-1990, Caregivers of children } \\
\text { 3-37 months with diarrhea in the } \\
\text { previous } 3 \text { days, } n=1496 \text { episodes }\end{array}$ & $\begin{array}{l}\text { Gave less cow's milk } \\
\text { than before diarrhea }\end{array}$ & 28.7 & & & & & & \\
\hline \multirow[t]{4}{*}{$\begin{array}{l}\text { Othero et al., } \\
2008 \text { [7] }\end{array}$} & \multirow[t]{4}{*}{ Kenya } & \multirow{4}{*}{$\begin{array}{l}\text { Longitudinal study, Nyanza Province } \\
2004-2006 \text {, Caregivers of children } \\
<5 \text { years in reference to most } \\
\text { recent episode, } n=927\end{array}$} & $\begin{array}{l}\text { Offered nothing } \\
\text { to drink }\end{array}$ & 20.5 & & & $\begin{array}{l}\text { Did not eat anything } \\
\text { (among all children) }\end{array}$ & 39 & $\begin{array}{l}\text { Gave anti-diarrheal } \\
\text { drugs }\end{array}$ & 45.3 \\
\hline & & & Offered much less ${ }^{\mathrm{e}}$ & 59.9 & & & & & & \\
\hline & & & $\begin{array}{l}\text { Offered somewhat } \\
\text { less }^{\mathrm{e}}\end{array}$ & 3.3 & & & & & & \\
\hline & & & Offered same $e^{e}$ & 5.3 & & & & & & \\
\hline \multirow{3}{*}{$\begin{array}{l}\text { Burton et al., } \\
2011 \text { [98] }\end{array}$} & \multirow[t]{3}{*}{ Kenya } & \multirow{3}{*}{$\begin{array}{l}\text { Cross-sectional survey, Rural Western } \\
\text { Kenya 2005, Caregivers of children } \\
<5 \text { years with diarrhea in the } \\
\text { previous } 2 \text { weeks, } n=188\end{array}$} & & & & & & & Gave antibiotic & 62.4 \\
\hline & & & & & & & & & Gave antimalarial & 52.4 \\
\hline & & & & & & & & & Gave IV fluid & 2.6 \\
\hline \multirow[t]{10}{*}{$\begin{array}{l}\text { Olson et al., } \\
2011[42]\end{array}$} & \multirow[t]{10}{*}{ Kenya } & \multirow[t]{10}{*}{$\begin{array}{l}\text { Cross-sectional survey, Asembo } \\
(n=371) \text { and Kibera }(n=389) \\
2007 \text {, Caregivers of children } \\
<5 \text { years with diarrhea in the } \\
\text { previous } 2 \text { weeks }\end{array}$} & $\begin{array}{l}\text { Asembo: Stopped } \\
\text { fluids other than } \\
\text { breast milk and } \\
\text { porridge (among } \\
\text { those giving fluids in } \\
\text { week before illness) }\end{array}$ & 9 & $\begin{array}{l}\text { Asembo: Stopped } \\
\text { breastfeeding }^{b}\end{array}$ & 5 & $\begin{array}{l}\text { Asembo: Stopped } \\
\text { porridge }\end{array}$ & 9 & $\begin{array}{l}\text { Asembo: Gave } \\
\text { oral medication } \\
\text { (not ORS or herbs) }\end{array}$ & 77 \\
\hline & & & $\begin{array}{l}\text { Kibera: Stopped fluids } \\
\text { other than breast milk } \\
\text { and porridge }\end{array}$ & 18 & $\begin{array}{l}\text { Kibera: Stopped } \\
\text { breastfeeding }\end{array}$ & 16 & $\begin{array}{l}\text { Kibera: Stopped } \\
\text { porridge }\end{array}$ & 36 & $\begin{array}{l}\text { Kibera: Gave } \\
\text { oral medication } \\
\text { (not ORS or herbs) }\end{array}$ & 81 \\
\hline & & & $\begin{array}{l}\text { Asembo: Decreased } \\
\text { fluidsh }\end{array}$ & 42 & $\begin{array}{l}\text { Asembo: Decreased } \\
\text { breastfeeding }\end{array}$ & 32 & $\begin{array}{l}\text { Asembo: Decreased } \\
\text { porridge }^{h}\end{array}$ & 54 & $\begin{array}{l}\text { Asembo: Gave } \\
\text { injected medication }\end{array}$ & 24 \\
\hline & & & $\begin{array}{l}\text { Kibera: Decreased } \\
\text { fluidsh }\end{array}$ & 47 & $\begin{array}{l}\text { Kibera: Decreased } \\
\text { breastfeedingh }\end{array}$ & 47 & $\begin{array}{l}\text { Kibera: Decreased } \\
\text { porridge }\end{array}$ & 69 & $\begin{array}{l}\text { Kibera: Gave } \\
\text { injected medication }\end{array}$ & 28 \\
\hline & & & Asembo: Same fluids ${ }^{h}$ & 47 & $\begin{array}{l}\text { Asembo: Same } \\
\text { breastfeeding }\end{array}$ & 59 & $\begin{array}{l}\text { Asembo: Same } \\
\text { porridge }\end{array}$ & 41 & $\begin{array}{l}\text { Asembo: Gave IV } \\
\text { fluids }\end{array}$ & 8 \\
\hline & & & Kibera: Same fluidsh & 22 & $\begin{array}{l}\text { Kibera: Same } \\
\text { breastfeeding }\end{array}$ & 28 & $\begin{array}{l}\text { Kibera: Same } \\
\text { porridge }^{h}\end{array}$ & 18 & Kibera: Gave IV fluids & 7 \\
\hline & & & & & & & $\begin{array}{l}\text { Asembo: Stopped } \\
\text { soft or solid food }\end{array}$ & 10 & & \\
\hline & & & & & & & $\begin{array}{l}\text { Kibera: Stopped soft } \\
\text { or solid food }\end{array}$ & 37 & & \\
\hline & & & & & & & $\begin{array}{l}\text { Asembo: Decreased } \\
\text { solid food }\end{array}$ & 54 & & \\
\hline & & & & & & & $\begin{array}{l}\text { Kibera: Decreased } \\
\text { solid food< }\end{array}$ & 70 & & \\
\hline
\end{tabular}


Table 1 Prevalence of harmful practices by region and country (Continued)

\begin{tabular}{|c|c|c|c|c|c|c|c|c|}
\hline & & & & & $\begin{array}{l}\text { Asembo: Same } \\
\text { solid food }\end{array}$ & 41 & & \\
\hline & & & & & $\begin{array}{l}\text { Kibera: Same } \\
\text { solid food }\end{array}$ & 23 & & \\
\hline & & & & & $\begin{array}{l}\text { Asembo: Stopped } \\
\text { or Decreased } \\
\text { feeding (including } \\
\text { BF, porridge, solids) }\end{array}$ & 36 & & \\
\hline & & & & & $\begin{array}{l}\text { Kibera: Stopped or } \\
\text { Decreased feeding } \\
\text { (including BF, } \\
\text { porridge, solids) }\end{array}$ & 54 & & \\
\hline \multirow[t]{5}{*}{$\begin{array}{l}\text { Omore et al., } \\
2013 \text { [41] }\end{array}$} & \multirow[t]{5}{*}{ Kenya } & \multirow{5}{*}{$\begin{array}{l}\text { Cross-sectional survey (HUAS), } \\
\text { Western Kenya 2007, Caregivers of } \\
\text { children }<5 \text { years with diarrhea in } \\
\text { the previous } 2 \text { weeks, } n=275\end{array}$} & $\begin{array}{l}\text { Offered same amount } \\
\text { to drink }\end{array}$ & 19 & $\begin{array}{l}\text { Offered usual } \\
\text { amount to eat }\end{array}$ & 16 & & \\
\hline & & & Offered less to drink & 67 & Offered less to eat & 83 & & \\
\hline & & & $\begin{array}{l}\text { Among those offering } \\
\text { less:Somewhat less }\end{array}$ & 52 & $\begin{array}{l}\text { Among offering } \\
\text { less:Somewhat less }\end{array}$ & 33 & & \\
\hline & & & Much less & 38 & Much less & 30 & & \\
\hline & & & Nothing & 10 & Nothing & 37 & & \\
\hline $\begin{array}{l}\text { Nasrin et al., } \\
2013 \text { [91] }\end{array}$ & Kenya & $\begin{array}{l}\text { Cross-sectional survey (HUAS), } \\
\text { Western Kenya 2007, Caregivers of } \\
\text { children }<5 \text { years with diarrhea in } \\
\text { the previous } 2 \text { weeks, } n=275\end{array}$ & & & & & $\begin{array}{l}\text { Gave leftover } \\
\text { antibiotics at home }\end{array}$ & 16 \\
\hline \multirow{2}{*}{$\begin{array}{l}\text { Zwisler et al., } \\
2013 \text { [68] }\end{array}$} & \multirow[t]{2}{*}{ Kenya } & \multirow{2}{*}{$\begin{array}{l}\text { Cross-sectional survey, } 4 \text { Provinces } \\
2012 \text {, Caregivers of children } \\
<5 \text { years with diarrhea in the } \\
\text { previous } 2 \text { months, } n=857\end{array}$} & & & & & Gave antibiotic & 51.3 \\
\hline & & & & & & & $\begin{array}{l}\text { Gave antimotility } \\
\text { agent }\end{array}$ & 10.4 \\
\hline \multirow[t]{4}{*}{$\begin{array}{l}\text { Simpson et al., } \\
2013 \text { [99] }\end{array}$} & \multirow[t]{4}{*}{ Kenya } & \multirow{4}{*}{$\begin{array}{l}\text { Cross-sectional survey, Western } \\
\text { Kenya (year not specified), } \\
\text { Caregivers of children } 6-60 \text { month } \\
\text { with diarrhea in the previous } \\
6 \text { months, } n=100\end{array}$} & & & & & $\begin{array}{l}\text { Gave antibiotic } \\
\text { (at any point) }\end{array}$ & 64 \\
\hline & & & & & & & $\begin{array}{l}\text { Gave antimotility } \\
\text { (at any point) }\end{array}$ & 13 \\
\hline & & & & & & & $\begin{array}{l}\text { Gave antibiotic } \\
\left(1^{\text {st }} \text { treatment }\right)\end{array}$ & 26 \\
\hline & & & & & & & $\begin{array}{l}\text { Gave antibiotic } \\
\text { ( } 1^{\text {st }} \text { or } 2^{\text {nd }} \text { treatment) }\end{array}$ & 46 \\
\hline
\end{tabular}


Table 1 Prevalence of harmful practices by region and country (Continued)

\begin{tabular}{|c|c|c|c|c|c|c|c|c|c|c|}
\hline \multirow[t]{6}{*}{$\begin{array}{l}\text { Winch et al., } \\
2008 \text { [71] }\end{array}$} & \multirow[t]{6}{*}{ Mali } & \multirow{6}{*}{$\begin{array}{l}\text { Cross-sectional baseline survey } \\
\text { preceding intervention, Southern } \\
\text { Mali 2004, Caregivers of children } \\
<5 \text { years with diarrhea in the } \\
\text { previous } 2 \text { weeks, } n=228\end{array}$} & $\begin{array}{l}\text { Same or less fluid or } \\
\text { breast milk }\end{array}$ & \multicolumn{5}{|l|}{82.7} & Gave antibiotics & 57 \\
\hline & & & $\begin{array}{l}\text { Stopped feeding or } \\
\text { breastfeeding }\end{array}$ & \multirow{5}{*}{\multicolumn{5}{|c|}{46}} & Gave metronidazole & 7.5 \\
\hline & & & & & & & & & Gave antidiarrheal & 2.6 \\
\hline & & & & & & & & & $\begin{array}{l}\text { Among children with } \\
\text { only diarrhea } \\
\text { symptoms gave: } \\
\text { Antibiotic }\end{array}$ & 16 \\
\hline & & & & & & & & & Antimalarial & 16 \\
\hline & & & & & & & & & Paracetamol & 10 \\
\hline $\begin{array}{l}\text { Perez et al., } \\
2009 \text { [100] }\end{array}$ & Mali & $\begin{array}{l}\text { Cross-sectional survey in } \\
\text { intervention comparison area, } \\
\text { Mopti Region 2006, Caregivers of } \\
\text { children }<5 \text { years, reference } \\
\text { episode unclear, } n=401\end{array}$ & & & & & & & Gave any drug & 56.1 \\
\hline $\begin{array}{l}\text { Nasrin et al., } \\
2013 \text { [91] }\end{array}$ & Mozambique & $\begin{array}{l}\text { Cross-sectional survey, Rural } \\
\text { Southern Mali 2007, Caregivers of } \\
\text { children }<5 \text { years with diarrhea in } \\
\text { the previous } 2 \text { weeks, } n=67\end{array}$ & & & & & $\begin{array}{l}\text { Offered less than } \\
\text { usual to eat }\end{array}$ & 38.3 & $\begin{array}{l}\text { Gave leftover } \\
\text { antibiotics at home }\end{array}$ & 3.6 \\
\hline \multirow[t]{4}{*}{$\begin{array}{l}\text { Nhampossa et al., } \\
2013 \text { [15] }\end{array}$} & \multirow[t]{4}{*}{ Mozambique } & \multirow{4}{*}{$\begin{array}{l}\text { Cross-sectional study (HUAS), Rural } \\
\text { Southern Mozambique } 2007 \\
\text { (Study } 1 n=67 \text { ) and 2009-2012 } \\
\text { (Study } 2 n=246 \text { ), Caregivers of } \\
\text { children }<5 \text { years with diarrhea in } \\
\text { previous } 2 \text { weeks }\end{array}$} & $\begin{array}{l}\text { Study 1: Reduced or } \\
\text { stopped breastfeeding/ } \\
\text { usual fluid intake }\end{array}$ & 12 & & & & & \multirow[t]{6}{*}{$\begin{array}{l}\text { Study 1: Gave } \\
\text { antibiotic (Among } \\
\text { those seeking } \\
\text { treatment) }\end{array}$} & \multirow[t]{6}{*}{14} \\
\hline & & & $\begin{array}{l}\text { Study 1: Maintained } \\
\text { same fluid or breast } \\
\text { milk intake }\end{array}$ & 73 & & & & & & \\
\hline & & & $\begin{array}{l}\text { Study 2: Reduced or } \\
\text { stopped breastfeeding/ } \\
\text { usual fluid intake }\end{array}$ & 79 & & & & & & \\
\hline & & & $\begin{array}{l}\text { Study 2: Maintained } \\
\text { same fluid or breast } \\
\text { milk intake }\end{array}$ & 1 & & & & & & \\
\hline \multirow[t]{2}{*}{$\begin{array}{l}\text { Ekanem et al., } \\
1990[47]\end{array}$} & \multirow[t]{2}{*}{ Nigeria } & \multirow{2}{*}{$\begin{array}{l}\text { Diarrhea surveillance survey, } \\
\text { Periurban Lagos (year not specified), } \\
\text { Mothers of children } 6-36 \text { months, } \\
\text { reference episode is general case, } \\
n=200\end{array}$} & & & $\begin{array}{l}\text { Normal breastfeeding } \\
\text { pattern continued }^{b}\end{array}$ & 76.9 & & & & \\
\hline & & & & & $\begin{array}{l}\text { Decreased } \\
\text { breastfeeding }^{b}\end{array}$ & 10.4 & & & & \\
\hline \multirow[t]{2}{*}{$\begin{array}{l}\text { Babaniyi et al., } \\
1994 \text { [10] }\end{array}$} & \multirow[t]{2}{*}{ Nigeria } & \multirow{2}{*}{$\begin{array}{l}\text { Cross-sectional study, Suleja 1991, } \\
\text { Caregivers of children }<5 \text { years } \\
\text { with diarrhea in previous } 2 \text { weeks, } \\
n=340\end{array}$} & $\begin{array}{l}\text { Normal amount of } \\
\text { "other" fluids"i }\end{array}$ & 55.6 & \multirow[t]{2}{*}{$\begin{array}{l}\text { Stopped } \\
\text { breastfeeding }\end{array}$} & \multirow[t]{2}{*}{7.7} & \multirow[t]{2}{*}{$\begin{array}{l}\text { Stopped or less } \\
\text { solid food }\end{array}$} & \multirow[t]{2}{*}{42.4} & \multirow[t]{2}{*}{$\begin{array}{l}\text { Gave any drug } \\
\text { (at home) }\end{array}$} & \multirow[t]{2}{*}{53.5} \\
\hline & & & Less "other" fluids" & 22.6 & & & & & & \\
\hline
\end{tabular}


Table 1 Prevalence of harmful practices by region and country (Continued)

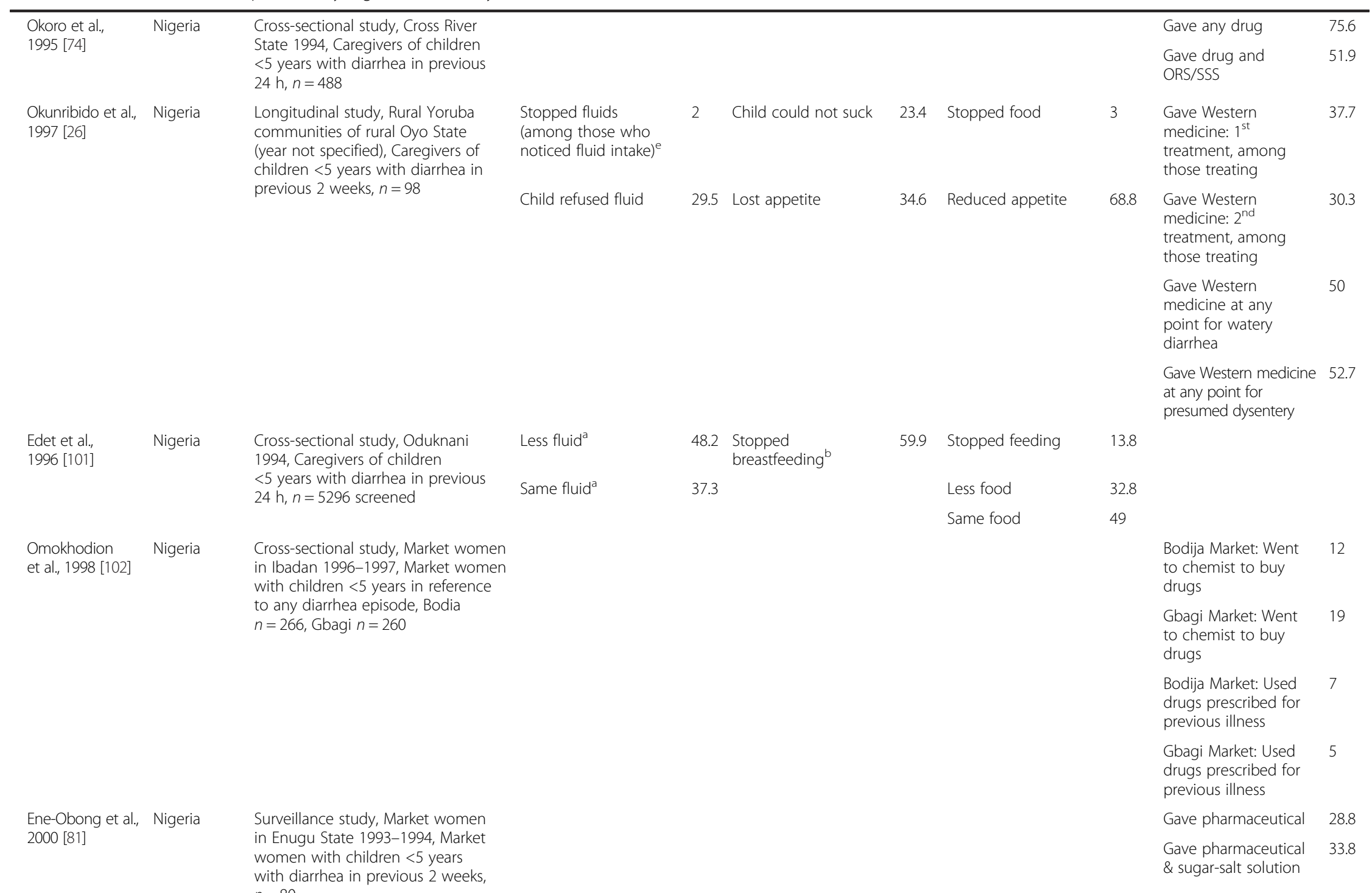


Table 1 Prevalence of harmful practices by region and country (Continued)

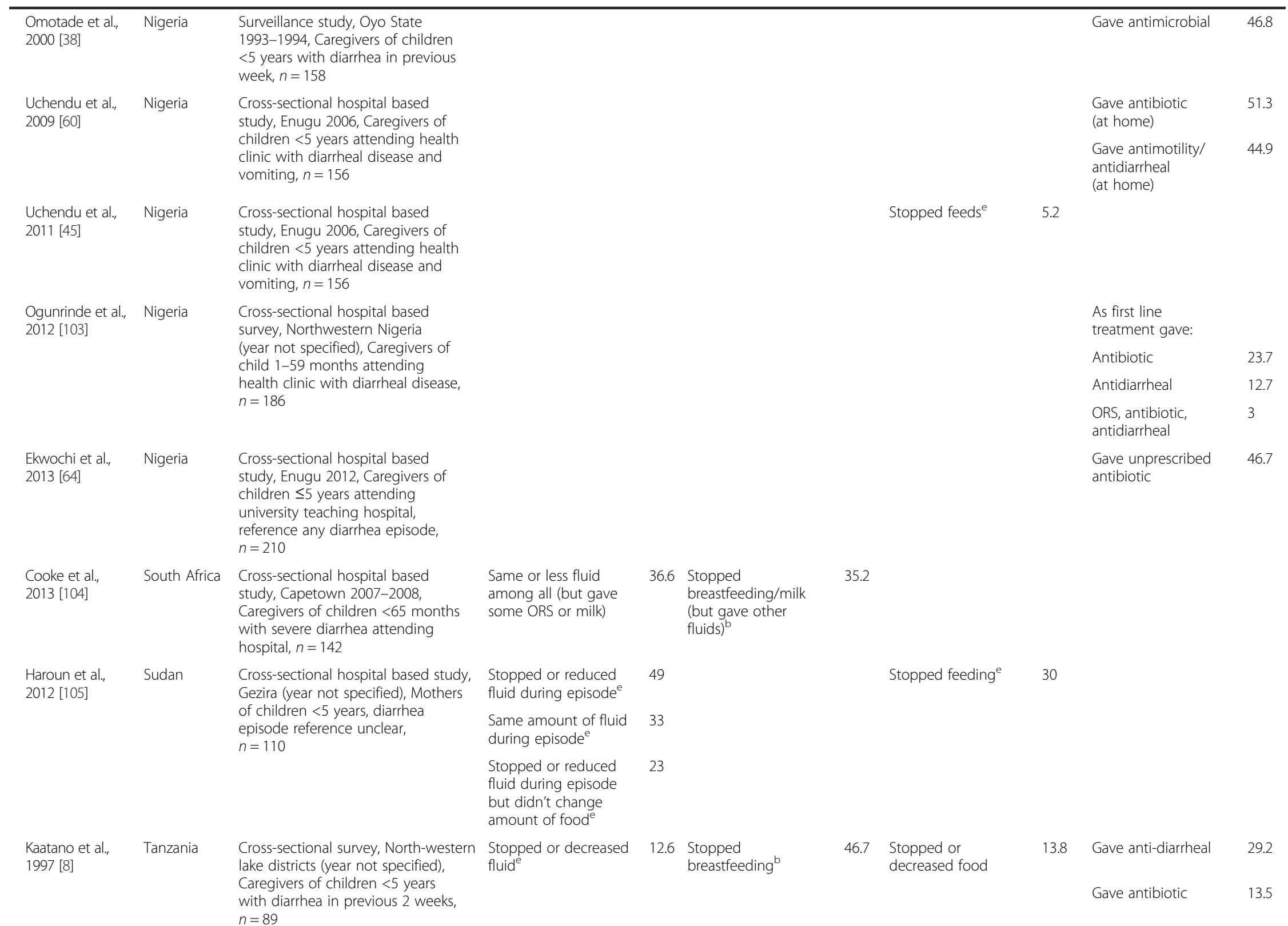


Table 1 Prevalence of harmful practices by region and country (Continued)

\begin{tabular}{|c|c|c|c|c|c|c|c|c|c|c|}
\hline South East Asia & & & & & & & & & & \\
\hline $\begin{array}{l}\text { Alam et al., } \\
1998 \text { [82] }\end{array}$ & Bangladesh & $\begin{array}{l}\text { Cross-sectional survey, Metropolitan } \\
\text { Chittagong 1996-1997, Caregivers } \\
\text { of children }<5 \text { years with diarrhea } \\
\text { in previous } 2 \text { weeks, } n=360\end{array}$ & & & & & & & $\begin{array}{l}\text { "Inappropriate or } \\
\text { non-recommended } \\
\text { drug use" among } \\
\text { those receiving } \\
\text { treatment }\end{array}$ & 73.5 \\
\hline & & & & & & & & & $\begin{array}{l}\text { Gave metronidazole } \\
\text { (denominator all } \\
\text { consultations) }\end{array}$ & 38.6 \\
\hline & & & & & & & & & $\begin{array}{l}\text { Gave antibiotic } \\
\text { (denominator } \\
\text { all consultations) }\end{array}$ & 17.5 \\
\hline & & & & & & & & & $\begin{array}{l}\text { Gave antiemetic } \\
\text { (denominator all } \\
\text { consultations) }\end{array}$ & 12.2 \\
\hline & & & & & & & & & $\begin{array}{l}\text { Gave antidiarrheal } \\
\text { (denominator all } \\
\text { consultations) }\end{array}$ & 8 \\
\hline $\begin{array}{l}\text { Ali et al., } \\
2000 \text { [27] }\end{array}$ & Bangladesh & $\begin{array}{l}\text { Cross-sectional survey, Brahmanharia } \\
\text { district 1993, Caregivers of children } \\
<5 \text { years with diarrhea in previous } \\
24 \mathrm{~h}, n=186\end{array}$ & $\begin{array}{l}\text { Drank less than usual } \\
\text { amount of water } \\
\text { (not amount offered) }\end{array}$ & 17 & & & & & & \\
\hline $\begin{array}{l}\text { Taha et al., } \\
2002 \text { [106] }\end{array}$ & Bangladesh & $\begin{array}{l}\text { Cross-sectional survey, Cox's Bazar } \\
\text { district 1994, Mothers of children } \\
<5 \text { years, diarrhea episode reference } \\
\text { unclear, } n=297\end{array}$ & $\begin{array}{l}\text { No fluids for treating } \\
\text { diarrhea }^{\text {e }}\end{array}$ & 11.7 & $\begin{array}{l}\text { Stopped } \\
\text { breastfeeding }^{b}\end{array}$ & 11.7 & $\begin{array}{l}\text { Did not give solid } \\
\text { or semi-solid food }^{c}\end{array}$ & 40.4 & & \\
\hline Baqui et al., & Bangladesh & Community based controlled trial, & & & & & & & Gave antibiotic & 34.3 \\
\hline 2004 [/3] & & $\begin{array}{l}\text { Matlab 1998-2000, Caregivers of } \\
\text { children 3-59 months with diarrhea }\end{array}$ & & & & & & & Gave other medicine & 44.8 \\
\hline & & in previous week, $n=297$ & & & & & & & Gave IV & 0.3 \\
\hline $\begin{array}{l}\text { Larson et al., } \\
2009 \text { [107] }\end{array}$ & Bangladesh & $\begin{array}{l}\text { Cross-sectional baseline survey } \\
\text { preceding intervention, Dhaka } \\
2006 \text {, Caregivers of children } \\
6-59 \text { months with diarrhea in } \\
\text { previous } 2 \text { weeks, } n=640\end{array}$ & & & & & & & Gave antibiotic & 34.7 \\
\hline $\begin{array}{l}\text { Das et al., } \\
2013 \text { [14] }\end{array}$ & Bangladesh & $\begin{array}{l}\text { Cross-sectional survey (HUAS), } \\
\text { Rural Mirzapur 2007, Caregivers of } \\
\text { children }<5 \text { years with diarrhea in }\end{array}$ & $\begin{array}{l}\text { Offered less than usual } \\
\text { amount of fluids }\end{array}$ & 10.8 & & & $\begin{array}{l}\text { Offered less to eat } \\
\text { (at home before } \\
\text { seeking care) }\end{array}$ & 28.7 & $\begin{array}{l}\text { Gave antibiotics } \\
\text { (at home before } \\
\text { seeking care) }\end{array}$ & 2.4 \\
\hline & & previous 2 weeks, $n=1128$ & Same amount & 61.3 & & & & & & \\
\hline & & & Same or less & 72.1 & & & & & & \\
\hline
\end{tabular}


Table 1 Prevalence of harmful practices by region and country (Continued)

\begin{tabular}{|c|c|c|c|c|c|c|c|c|c|c|}
\hline $\begin{array}{l}\text { Sood et al., } \\
1990 \text { [108] }\end{array}$ & India & $\begin{array}{l}\text { Cross-sectional survey, Rural Haryana } \\
\text { State (year not specified), Caregivers } \\
\text { of children }<5 \text { years, reference any } \\
\text { diarrhea episode, } n=108\end{array}$ & & & $\begin{array}{l}\text { Generally stopped } \\
\text { breastfeeding }\end{array}$ & 0 & $\begin{array}{l}\text { Some food } \\
\text { restricted }\end{array}$ & 83.33 & & \\
\hline \multirow[t]{4}{*}{$\begin{array}{l}\text { Rasania et al., } \\
1993 \text { [23] }\end{array}$} & \multirow[t]{4}{*}{ India } & \multirow{4}{*}{$\begin{array}{l}\text { Cross-sectional survey, New Delhi } \\
\text { (year not specified), Caregivers of } \\
\text { children }<5 \text { years, diarrhea episode } \\
\text { reference unclear, } n=254\end{array}$} & & & $\begin{array}{l}\text { Restricted } \\
\text { breastfeeding }\end{array}$ & 12.59 & $\begin{array}{l}\text { Gave less food during } \\
\text { convalescence }\end{array}$ & 26.38 & & \\
\hline & & & & & $\begin{array}{l}\text { Stopped } \\
\text { breastfeeding }^{\text {b }}\end{array}$ & 19.29 & $\begin{array}{l}\text { Shifted from solid } \\
\text { to liquid diet }\end{array}$ & 45.27 & & \\
\hline & & & & & & & Stopped all food & 9.84 & & \\
\hline & & & & & & & $\begin{array}{l}\text { Restricted } \\
\text { "few" foods }\end{array}$ & 16.53 & & \\
\hline $\begin{array}{l}\text { Gupta et al., } \\
2007 \text { [109] }\end{array}$ & India & $\begin{array}{l}\text { Cross-sectional survey, Urban Delhi } \\
\text { slum 2004, Caregivers of children } \\
<5 \text { years with diarrhea in previous } \\
2 \text { weeks, } n=\text { unclear } 1307\end{array}$ & Stopped fluide & 20 & & & $\begin{array}{l}\text { Stopped feeding } \\
\text { (not clear if food } \\
\text { or breastfeeding) }\end{array}$ & 50 & & \\
\hline \multirow[t]{2}{*}{$\begin{array}{l}\text { Ahmed et al., } \\
2009 \text { [46] }\end{array}$} & \multirow[t]{2}{*}{ India } & \multirow{2}{*}{$\begin{array}{l}\text { Cross-sectional survey, Kashmir } \\
\text { Valley 2006, Caregivers of children } \\
<5 \text { years with diarrhea in previous } \\
24 \mathrm{~h}(n=1055) \text { and } 2 \text { weeks } \\
(n=2836)\end{array}$} & & & & & $\begin{array}{l}\text { Among diarrhea } \\
\text { in } 15 \text { days: Feeding } \\
\text { restricted }\end{array}$ & 4 & $\begin{array}{l}\text { Diarrhea in last } 24 \mathrm{~h} \text { : } \\
\text { Gave antibiotic }\end{array}$ & 77.9 \\
\hline & & & & & & & $\begin{array}{l}\text { Diarrhea in last } 24 \mathrm{~h} \text { : } \\
\text { Feeding restricted }^{\mathrm{e}}\end{array}$ & 6.9 & & \\
\hline \multirow[t]{2}{*}{$\begin{array}{l}\text { Shah et al., } \\
2012 \text { [31] }\end{array}$} & \multirow[t]{2}{*}{ India } & \multirow[t]{2}{*}{$\begin{array}{l}\text { Cross-sectional survey, Urban slum } \\
\text { of Aligarh 2009, Caregivers of } \\
\text { children }<5 \text { years with diarrhea in } \\
\text { previous } 2 \text { weeks, } n=101\end{array}$} & & & $\begin{array}{l}\text { Stopped or decreased } \\
\text { breastfeeding (among } \\
\text { EBF 0-6 m) }\end{array}$ & 30.77 & $\begin{array}{l}\text { Interrupted, stopped } \\
\text { or decreased } \\
\text { feeding (among } \\
\text { not breastfeeding: } \\
7 \mathrm{~m}-5 \text { years) }\end{array}$ & 37.8 & & \\
\hline & & & & & $\begin{array}{l}\text { Stopped or decreased } \\
\text { breastfeeding (among } \\
\text { non-EBF } 0-6 \mathrm{~m})^{\mathrm{b}}\end{array}$ & 80 & & & & \\
\hline \multirow{2}{*}{$\begin{array}{l}\text { Zwisler et al., } \\
2013 \text { [68] }\end{array}$} & \multirow[t]{2}{*}{ India } & \multirow{2}{*}{\multicolumn{2}{|c|}{$\begin{array}{l}\text { Cross-sectional survey, } 7 \text { States } 2012 \text {, } \\
\text { Caregivers of children }<5 \text { years } \\
\text { with diarrhea in the previous } \\
2 \text { months, } n=988\end{array}$}} & & & & & & Gave antibiotic & 56.4 \\
\hline & & & & & & & & & $\begin{array}{l}\text { Gave antimotility } \\
\text { agent }\end{array}$ & 3 \\
\hline \multirow[t]{4}{*}{ WHO 1991 [110] } & \multirow[t]{4}{*}{ Nepal } & \multirow{4}{*}{$\begin{array}{l}\text { Cross-sectional survey, Terai }(n=335) \\
\text { and Midhills }(n=526) 1990 \text {, } \\
\text { Caregivers of children }<5 \text { years with } \\
\text { diarrhea in previous } 24 \mathrm{~h}\end{array}$} & $\begin{array}{l}\text { Terai: Same or } \\
\text { less fluid }^{\mathrm{a}}\end{array}$ & 72 & $\begin{array}{l}\text { Terai: Stopped } \\
\text { breastfeeding }^{\text {b }}\end{array}$ & 1 & $\begin{array}{l}\text { Terai: Stopped or } \\
\text { Less Feeding }\end{array}$ & 25 & $\begin{array}{l}\text { Terai: Gave drug, } \\
\text { no ORS }\end{array}$ & 21.5 \\
\hline & & & $\begin{array}{l}\text { Midhills: Same or } \\
\text { less fluid }^{\mathrm{a}}\end{array}$ & 91 & $\begin{array}{l}\text { Midhills: Stopped } \\
\text { breastfeeding }\end{array}$ & 1 & $\begin{array}{l}\text { Midhills: Stopped } \\
\text { or Less Feeding }\end{array}$ & 39 & $\begin{array}{l}\text { Midhills: Gave drug, } \\
\text { no ORS }\end{array}$ & 14.3 \\
\hline & & & & & & & & & $\begin{array}{l}\text { Terai: Gave drug } \\
\text { and ORS }\end{array}$ & 4.5 \\
\hline & & & & & & & & & $\begin{array}{l}\text { Midhills: Gave drug } \\
\text { and ORS }\end{array}$ & 4.9 \\
\hline
\end{tabular}


Table 1 Prevalence of harmful practices by region and country (Continued)

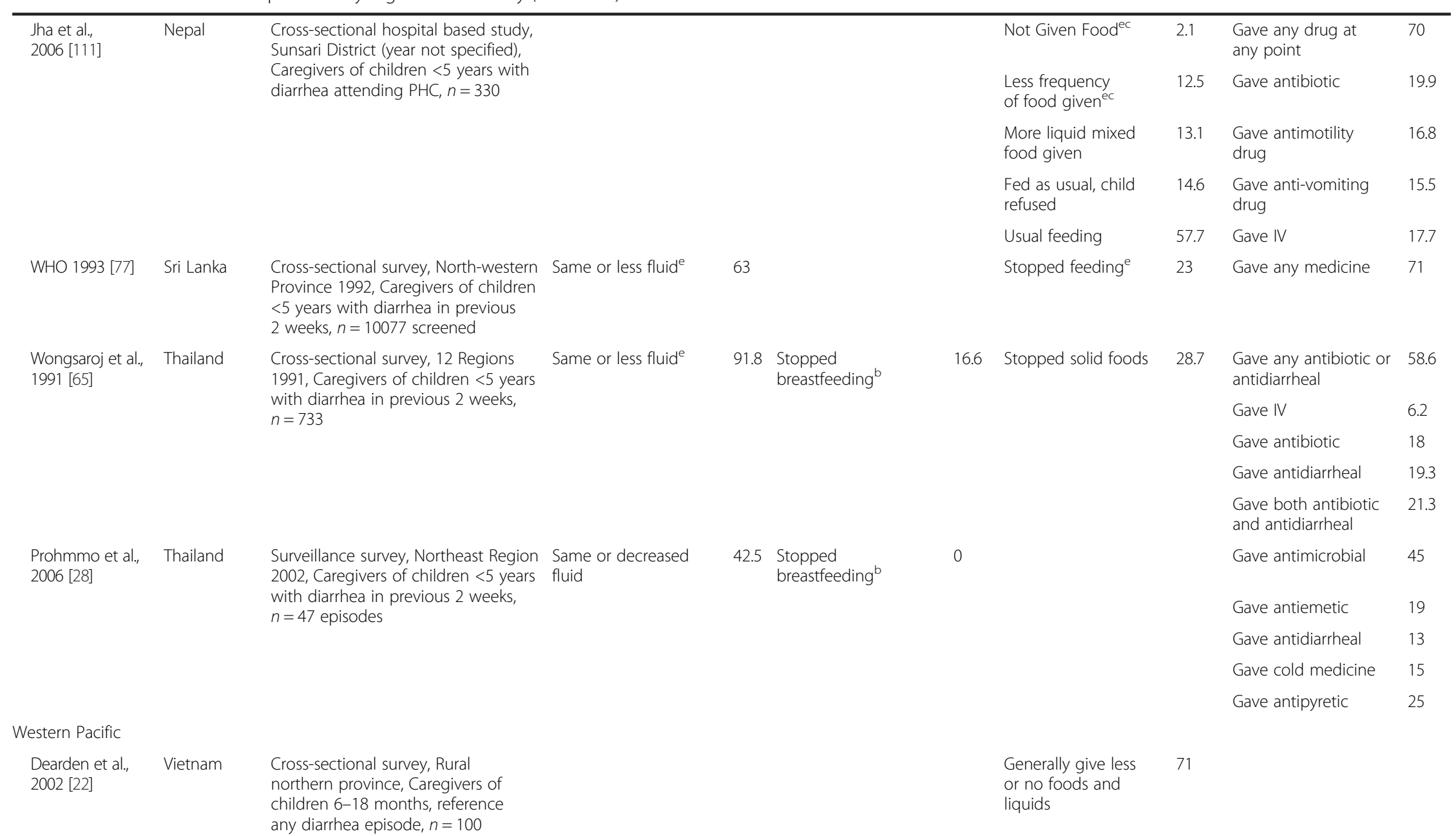


Table 1 Prevalence of harmful practices by region and country (Continued)

\begin{tabular}{lll}
\hline Hoan et al., & Vietnam & Cross-sectional survey, Rural district \\
(year not specified), Caregivers of \\
children $<5$ years with diarrhea in \\
previous 2 weeks, $n=133$
\end{tabular}

previous 2 weeks, $n=133$

Among children with 54.1

only diarrhea

symptoms gave:

Antibiotics

Anti-diarrheal

Antihistamine

Analgesic/antipyretic

${ }^{a}$ Excluding breast milk

${ }^{\mathrm{b}}$ Among those breastfeeding

'Unclear if only among those receiving solid or semi-solid food before illness

${ }^{\mathrm{d}}$ Among drinking fluids other than breast milk

eInclusion/exclusion of breastfeeding not specified

${ }^{\mathrm{f}}$ Among those receiving drug as treatment

IUnclear if only among those breastfeeding at time of illness

${ }^{h}$ Among those who continued to receive fluids; breast milk; food

'Explicitly excluding ORS/SSS 
Table 2 Factors associated with harmful practice

\begin{tabular}{|c|c|c|c|c|c|}
\hline Level & Factor & $\begin{array}{c}\text { Positive association } \\
\text { (harmful practice more likely) }\end{array}$ & $\begin{array}{c}\text { Negative association } \\
\text { (harmful practice less likely) }\end{array}$ & No association & No test of significance \\
\hline \multicolumn{6}{|c|}{ Association with fluid curtailment } \\
\hline \multirow[t]{5}{*}{ Episode } & Dehydrated (vs not dehydrated) & & & & {$[57]$} \\
\hline & Severe disease & [10] & & & {$[57]$} \\
\hline & Child vomited (vs did not vomit) & [9] & & & \\
\hline & Child was anorexic & & [11] & & \\
\hline & Longer duration of episode & & [11] & & \\
\hline Child & Older child age & & [15] & {$[12]$} & {$[13,14]$} \\
\hline \multirow[t]{4}{*}{ Caregiver } & Older maternal age & & {$[12]$} & {$[16]$} & \\
\hline & Higher maternal education & & {$[16]$} & {$[12]$} & \\
\hline & Older maternal age at marriage & & & {$[12]$} & \\
\hline & Caregiver employed & & [12] & & \\
\hline Household & Live in urban area (vs rural) & & & [16] & [95] \\
\hline \multicolumn{6}{|c|}{ Association with breastfeeding restriction } \\
\hline \multirow[t]{2}{*}{ Episode } & Dehydrated (vs not dehydrated) & & & & [57] \\
\hline & Severe disease & & & & {$[57]$} \\
\hline Child & Older child age & & & {$[12]$} & \\
\hline \multirow[t]{4}{*}{ Caregiver } & Older maternal age & & [12] & & \\
\hline & Higher maternal education & & {$[12]$} & & \\
\hline & Older maternal age at marriage & & & {$[12]$} & \\
\hline & Caregiver employed & & & {$[12]$} & \\
\hline Household & Live in urban area (vs rural) & & & {$[33]$} & [95] \\
\hline \multicolumn{6}{|c|}{ Association with Food Restriction } \\
\hline \multirow[t]{6}{*}{ Episode } & Dehydrated (vs not dehydrated) & {$[40]$} & & & {$[57]$} \\
\hline & Severe disease & {$[40]$} & & & {$[57]$} \\
\hline & Child had fever & [11] & & & \\
\hline & Child was anorexic & [11] & & & \\
\hline & ORS use & [41] & & & \\
\hline & Sought care outside home & [41] & & & \\
\hline Child & Older child age & {$[42]$} & & {$[12]$} & {$[13,14]$} \\
\hline \multirow[t]{4}{*}{ Caregiver } & Older maternal age & & & {$[12,16]$} & [90] \\
\hline & Higher maternal education & & {$[12,16]$} & & {$[90]$} \\
\hline & Older maternal age at marriage & & & {$[12]$} & \\
\hline & Caregiver employed & & & {$[12]$} & \\
\hline \multirow[t]{2}{*}{ Household } & Greater household income & & & & [90] \\
\hline & Live in urban area (vs rural) & & & {$[16]$} & {$[90,95]$} \\
\hline \multicolumn{6}{|c|}{ Association with inappropriate drug use } \\
\hline \multirow[t]{6}{*}{ Episode } & Dehydrated (vs not dehydrated) & {$[60]$} & & [40] & {$[57]$} \\
\hline & Severe disease & & & {$[10,40]$} & [57] \\
\hline & Longer disease duration & [63] & & & \\
\hline & Classification of diarrhea & & & & [81] \\
\hline & ORS use & {$[60,63]$} & & & [68] \\
\hline & Sought care outside home & {$[11,41]$} & & & \\
\hline
\end{tabular}


Table 2 Factors associated with harmful practice (Continued)

\begin{tabular}{llcc}
\hline Child & Older child age & {$[13,14]$} \\
Caregiver & Higher maternal education & {$[64]$} & {$[60]$} \\
Household & Greater household income & {$[60,87]$} \\
& Live in urban area (vs rural) & {$[93]$} \\
\hline
\end{tabular}

[24]. The beliefs of family and community members, particularly elderly relatives, have also been reported as influential in determining caregiver practices related to fluids and feeding during childhood diarrhea episodes [22, 24, 25]. In three studies caregivers reported reduced fluid intake due to child refusal, child crying, or decreased thirst [22, 26, 27]. In one study, mothers reported they did not encourage increased fluids because they were inexperienced in how to do this [27].

\section{Breastfeeding reduction}

Many studies reported the practice of breastfeeding reduction or cessation during diarrhea episodes (Table 1, Column 5). Most studies found that among mothers breastfeeding their child prior to the onset of diarrhea, fewer than $10 \%$ of mothers stopped breastfeeding during the episode. The practice of breastfeeding cessation ranged from no mothers reporting breastfeeding cessation in a surveillance study in northeast Thailand to $62 \%$ of mothers reporting stopping breast or milk feeding in a hospital-based study in Saudi Arabia [20, 28]. The practice of breastfeeding cessation was higher in hospital samples compared to samples from the general population. Where breastfeeding reduction was reported, on average one quarter of mothers reported reducing breastfeeding, although there was significant variation in the practice.

Multiple studies assessed variance in breastfeeding restriction by factors including characteristics of the diarrhea episode, child, caregiver, and household (Table 2). One study found younger and less educated mothers were more likely to reduce breastfeeding during episodes of diarrhea [12].

Mothers reported ceasing or reducing breastfeeding when their child had diarrhea for various reasons (Table 3). Mothers reported stopping or reducing breastfeeding because of beliefs that breastmilk was too fatty to be digested [20]. Others reported continued breastfeeding would not reduce the duration of diarrhea [20,29] or could cause or worsen the diarrhea $[18,19,29]$. Caregivers in two studies believed specific types of diarrhea must be treated with breastfeeding cessation [29, 30]. In multiple cultures, "dirty" breast milk or secretion of ingested food through breast milk was thought to cause certain types of diarrhea. Mothers received treatment or a modified diet to improve the quality of their breast milk [31-34] or children were weaned [35]. Some caregivers stated they were following the advice of healthcare providers by restricting breastfeeding [20, 36]. Older relatives were also important sources of information on feeding practices during diarrhea episodes [25, 31]. In some studies, mothers continued feeding but diluted milk or formula [29], switched to powdered or goat's milk [37], or only gave water [38].

\section{Food restriction}

The measurement of food restriction, and prevalence estimates, varied widely across studies (Table 1, Column 6). Many studies differed in their definition or failed to specify if food restriction was measured only among those eating solid foods prior to illness, whether breastfeeding was included or excluded, and whether amount of food offered versus consumed was measured. Findings on restriction of specific foods have been included for context but not in prevalence estimates of overall food restriction (Table 1). The practice of stopping all food ranged from as low as $3 \%$ of mothers stating they stopped giving solid or semi-solid foods during the episode in Oyo State, Nigeria [26] to as high as $53 \%$ of mothers reporting they stopped feeding in Kenya [39]. As expected, measures that included the reduction of feeding in addition to complete restriction of feeding showed higher rates of food restriction, mostly within the range of 30-60\% of episodes.

Multiple studies addressed the variance of food restriction by other factors, including characteristics of the diarrhea episode, child, caregiver, and household (Table 2). Food curtailment was associated with dehydration and more severe disease [40], seeking care outside of the home, and ORS use [41]. In one study, caregivers were more likely to withhold food if a child had fever or a low appetite [11]. Another study found children less than 2 years of age were more likely to receive continued feeding compared to older children [42]. Two studies found that less educated mothers were more likely to restrict foods $[12,16]$.

Motivation for food restriction differed (Table 3). Some caregivers reported that a child's diet should be restricted because of beliefs that a child cannot eat or digest as much during a diarrhea episode [22, 43] and feeding can exacerbate or prolong diarrhea episodes $[19,22,29,44-46]$. Belief that only certain foods should be restricted because they can aggravate diarrhea was common across countries and included a range of foods such as meat, milk, sweet food, greasy food, high 
Table 3 Beliefs, motivations, and context related to harmful practices by region and country

\begin{tabular}{|c|c|c|c|c|c|c|c|c|c|}
\hline $\begin{array}{l}\text { Author, } \\
\text { Year [reference] }\end{array}$ & Country & $\begin{array}{l}\text { Study design: methods } \\
\text { (number conducted), } \\
\text { study population }\end{array}$ & $\begin{array}{l}\text { Source of } \\
\text { information on } \\
\text { diarrhea treatment }\end{array}$ & $\begin{array}{l}\text { Expected effect } \\
\text { of treatment }\end{array}$ & $\begin{array}{l}\text { Restriction of specific } \\
\text { food or fluid }\end{array}$ & $\begin{array}{l}\text { Treatment specific } \\
\text { to type or cause } \\
\text { of diarrhea }\end{array}$ & $\begin{array}{l}\text { Drug specific: } \\
\text { strength/ } \\
\text { effectiveness }\end{array}$ & $\begin{array}{l}\text { Drug specific: } \\
\text { and source/ } \\
\text { availability }\end{array}$ & Other \\
\hline \multicolumn{10}{|l|}{ Americas } \\
\hline \multirow[t]{2}{*}{$\begin{array}{l}\text { Hudelson et al., } \\
1994 \text { [44] }\end{array}$} & \multirow[t]{2}{*}{ Bolivia } & \multirow{2}{*}{$\begin{array}{l}\text { Qualitative study: } \\
\text { Indepth interviews IDIs } \\
\text { (65), hypothetical case } \\
\text { scenarios (10), and } \\
\text { observation (5) of } \\
\text { mother and health } \\
\text { workers, El Alto 1993, } \\
\text { Mothers of children } \\
<5 \text { years and health } \\
\text { workers }\end{array}$} & & $\begin{array}{l}\text { Food: Mothers } \\
\text { worry increasing } \\
\text { food intake could } \\
\text { worsen episode }\end{array}$ & & $\begin{array}{l}\text { General: Type of } \\
\text { treatment sought } \\
\text { is dependent on } \\
\text { perceived cause } \\
\text { of the illness }\end{array}$ & & & $\begin{array}{l}\text { Feeding: Diet } \\
\text { is already poor } \\
\text { so doesn't vary } \\
\text { much during } \\
\text { episode }\end{array}$ \\
\hline & & & & $\begin{array}{l}\text { Food: Some } \\
\text { may offer } \\
\text { less food to } \\
\text { reduce stool } \\
\text { output }\end{array}$ & & $\begin{array}{l}\text { Drugs: Drugs are } \\
\text { used to treat } \\
\text { "diarrea por } \\
\text { infeccion" }\end{array}$ & & & $\begin{array}{l}\text { Food: } \\
\text { Reduction in } \\
\text { intake due to } \\
\text { loss of appetite. } \\
\text { Caregivers } \\
\text { unaccustomed } \\
\text { to encouraging } \\
\text { feeding. }\end{array}$ \\
\hline $\begin{array}{l}\text { Larrea-Killinger } \\
\text { et al., } 2013 \text { [113] }\end{array}$ & Brazil & $\begin{array}{l}\text { Qualitative study: IDIs } \\
\text { (29) and observations, } \\
\text { Salvador 1997-2004, } \\
\text { Mothers and } \\
\text { grandmothers of } \\
\text { children < } 5 \text { years }\end{array}$ & & & & & $\begin{array}{l}\text { Combination } \\
\text { of ORS and } \\
\text { antibiotics } \\
\text { believed to } \\
\text { reduce severity } \\
\text { of episode }\end{array}$ & & \\
\hline \multirow[t]{4}{*}{$\begin{array}{l}\text { McLennan et al., } \\
2002 \text { [49] }\end{array}$} & \multirow[t]{4}{*}{ Brazil } & \multirow{4}{*}{$\begin{array}{l}\text { Qualitative study: IDIs } \\
\text { (29) and observations, } \\
\text { Salvador 1997-2004, } \\
\text { Mothers and } \\
\text { grandmothers of } \\
\text { children < } 5 \text { years }\end{array}$} & & & $\begin{array}{l}\text { Feeding: } 1 / 3 \text { mothers } \\
\text { reported restricting } \\
\text { some foods }\end{array}$ & & & & \multirow{4}{*}{$\begin{array}{l}\text { Drugs: } 73 \% \\
\text { mothers } \\
\text { believe child } \\
\text { should be } \\
\text { given antibiotic } \\
\text { for episode }\end{array}$} \\
\hline & & & & & $\begin{array}{l}\text { Feeding: } 95 \% \\
\text { believe at least } \\
\text { one food item } \\
\text { should be restricted }\end{array}$ & & & & \\
\hline & & & & & $\begin{array}{l}\text { Food: } 38 \% \text { believe all } \\
\text { solid foods should } \\
\text { be restricted }\end{array}$ & & & & \\
\hline & & & & & $\begin{array}{l}\text { BF: Few (3 \%) } \\
\text { believe BF should } \\
\text { be suspended }\end{array}$ & & & & \\
\hline $\begin{array}{l}\text { Granich et al., } \\
1999 \text { [114] }\end{array}$ & $\begin{array}{l}\text { Dominican } \\
\text { Republic }\end{array}$ & $\begin{array}{l}\text { Quantitative study: } \\
\text { Structured interviews } \\
\text { (582), Periurban Santo } \\
\text { Domingo 1996, } \\
\text { Mothers of children } \\
<5 \text { years }\end{array}$ & & & & & & & $\begin{array}{l}\text { Drugs: } 71 \% \text { of } \\
\text { caregivers } \\
\text { would give pill } \\
\text { or injection for } \\
\text { hypothetical } \\
\text { episode of } \\
\text { diarrhea }\end{array}$ \\
\hline
\end{tabular}

Feeding: 1/3 mothers eported restricting

Feeding: $95 \%$

believe at least olid foods should

believe BF should

be suspended 
Table 3 Beliefs, motivations, and context related to harmful practices by region and country (Continued)

\begin{tabular}{|c|c|c|c|c|c|}
\hline $\begin{array}{l}\text { Ecker et al., } \\
2013 \text { [115] }\end{array}$ & Peru & $\begin{array}{l}\text { Quantitative study: } \\
\text { Structured interviews } \\
\text { (1200), Periurban Lima } \\
\text { (year not specified), } \\
\text { Caregivers of children } \\
<5 \text { years }\end{array}$ & & & $\begin{array}{l}\text { Drugs: } 65 \% \\
\text { of caregivers } \\
\text { believe } \\
\text { antibiotic is } \\
\text { necessary to } \\
\text { treat } \\
\text { hypothetical } \\
\text { case of non- } \\
\text { dysenteric } \\
\text { diarrhea }\end{array}$ \\
\hline \multicolumn{6}{|l|}{ Europe } \\
\hline \multicolumn{6}{|l|}{$\begin{array}{l}\text { Eastern } \\
\text { Mediterranean }\end{array}$} \\
\hline \multirow[t]{2}{*}{$\begin{array}{l}\text { Ali et al., } \\
2003 \text { [50] }\end{array}$} & \multirow[t]{2}{*}{ Pakistan } & \multirow{2}{*}{$\begin{array}{l}\text { Quantitative study: } \\
\text { Self-administered } \\
\text { questionnaire (400), } \\
\text { Karachi 2000, Adult } \\
\text { females attending } \\
\text { clinic }\end{array}$} & \multirow{4}{*}{$\begin{array}{l}\text { Food: Most } \\
\text { caregivers reported } \\
\text { receiving } \\
\text { information on } \\
\text { food restriction } \\
\text { from mother or } \\
\text { grandmother }\end{array}$} & $\begin{array}{l}\text { Food: Heavy foods, } \\
\text { bread, meat } \\
\text { commonly restricted }\end{array}$ & \\
\hline & & & & $\begin{array}{l}\text { Food: } 2 \% \text { of women } \\
\text { believe all food } \\
\text { items should be } \\
\text { restricted }\end{array}$ & \\
\hline \multirow[t]{2}{*}{$\begin{array}{l}\text { Agha et al., } \\
2007 \text { [116] }\end{array}$} & \multirow[t]{2}{*}{ Pakistan } & \multirow{2}{*}{$\begin{array}{l}\text { Quantitative study: } \\
\text { Structured interview } \\
\text { (647), Gambat, Singh } \\
\text { Province (year not } \\
\text { specified), Caregivers } \\
\text { of children 6-59 } \\
\text { months }\end{array}$} & & $\begin{array}{l}\text { Fluid: } 12 \% \text { of } \\
\text { caregivers believe } \\
\text { less fluid is required } \\
\text { during episode }\end{array}$ & \\
\hline & & & & $\begin{array}{l}\text { Food: } 44 \% \text { believe } \\
\text { less food is required }\end{array}$ & \\
\hline \multirow[t]{2}{*}{$\begin{array}{l}\text { Rasheed et al., } \\
1993 \text { [117] }\end{array}$} & \multirow[t]{2}{*}{$\begin{array}{l}\text { Saudi } \\
\text { Arabia }\end{array}$} & \multirow{2}{*}{$\begin{array}{l}\text { Quantitative study: } \\
\text { Structured interview } \\
\text { (240) and self- } \\
\text { administered } \\
\text { questionnaire (589), } \\
\text { Eastern Province 1990, } \\
\text { Mothers of children } \\
\text { attending government } \\
\text { health center and } \\
\text { girls attending } \\
\text { government high } \\
\text { school }\end{array}$} & & & $\begin{array}{l}\text { Feeding: Fewer } \\
\text { mothers than } \\
\text { female students } \\
\text { believe fluid } \\
\text { and foods } \\
\text { should be } \\
\text { restricted } \\
\text { during episode }\end{array}$ \\
\hline & & & & & $\begin{array}{l}\text { Drugs: } \\
\text { Compared to } \\
\text { students, } \\
\text { more mothers } \\
\text { preferred } \\
\text { drugs as } \\
\text { treatment }\end{array}$ \\
\hline
\end{tabular}


Table 3 Beliefs, motivations, and context related to harmful practices by region and country (Continued)

Afric

Kaltenthaler
et al., $1996[30]$

Botswana

Qualitative study:

Focus group

discussions FGDs (4)

and observations,

North-east Botswana

of young children,

health providers and

traditional healers

\begin{tabular}{|c|c|c|}
\hline $\begin{array}{l}\text { Nkwi et al., } \\
1994 \text { [34] }\end{array}$ & Cameroon & $\begin{array}{l}\text { Mixed-method study: } \\
\text { Structured interviews } \\
(256) \text { and hospital } \\
\text { observations, } 3 \\
\text { provinces in Cameroon, } \\
\text { Caregivers of children } \\
<5 \text { years }\end{array}$ \\
\hline
\end{tabular}

Almroth et al., Lesotho $1997[36]$ and IDIs (12), Klls (7)
1991-1992, Caregivers

BF: Pogwana

(severe diarrhea

with sunken

fontanel) is an

"African illness"

and should be

treated with

breast feeding

cessation and

should go to

health facility or

traditional healer

BF: Some diarrhea

thought to be

caused by "bad

breastmilk" -

herbs to improve

quality of milk

Food: Believe Food: Believe you food should be should adjust diet

$\begin{array}{ll}\text { (19) and IDIs (43), } & \text { received conflicting }\end{array}$ different locations

1991-1992, Mothers

and grandmothers

of children and nurses advice from

grandmothers and

nurses

given because it

"strengthens the

for individual child,

Feeding: Caregivers

report providers still

restrict feeding mothers are given

advise caregivers to

General:

Mothers report

using multiple

sources of

treatment if

episode

doesn't

improve

Food: Mothers coax children to eat during

and after

diarrhea

General:

Mothers report

using any

treatment that

works,

sometimes

multiple

treatments

BF: Perceived

causes of diarrhea

Drugs

and Klls (sample size

not specified), Rumphi

2000-2002, Old and

young men and

women and health

providers include

contaminated

breast milk

requires weaning

General: Diarrhea

due to teething

is perceived as

requiring no

treatment 
Table 3 Beliefs, motivations, and context related to harmful practices by region and country (Continued)

\begin{tabular}{|c|c|c|c|c|c|c|c|c|c|}
\hline $\begin{array}{l}\text { Ellis et al., } \\
2007 \text { [78] }\end{array}$ & Mali & $\begin{array}{l}\text { Mixed methods study: } \\
\text { Structured interviews } \\
\text { (352), illness narratives } \\
\text { (14), and IDIs (42), } \\
\text { Bougouni District } \\
\text { 2003, Caregivers of } \\
\text { children <5 years with } \\
\text { illness in past } 2 \text { weeks } \\
\text { or seeking care and } \\
\text { health providers }\end{array}$ & $\begin{array}{l}\text { General: } \\
\text { Mothers-in-law play } \\
\text { important role } \\
\text { initiating traditional } \\
\text { treatment }\end{array}$ & & & & $\begin{array}{l}\text { Combining } \\
\text { several } \\
\text { different } \\
\text { medicines/ } \\
\text { therapies is } \\
\text { viewed as } \\
\text { most } \\
\text { efficacious }\end{array}$ & $\begin{array}{l}\text { Treatment of } \\
\text { diarrhea } \\
\text { typically } \\
\text { begins in the } \\
\text { home with } \\
\text { traditional } \\
\text { medicines } \\
\text { and/or } \\
\text { antibiotics } \\
\text { from nearby } \\
\text { vendors }\end{array}$ & \\
\hline \multirow[t]{3}{*}{$\begin{array}{l}\text { Ikpatt et al., } \\
1992 \text { [19] }\end{array}$} & \multirow[t]{3}{*}{ Nigeria } & \multirow{3}{*}{$\begin{array}{l}\text { Quantitative study: } \\
\text { Self-administered } \\
\text { questionnaire (561), } \\
\text { Cross River and Akwa } \\
\text { Iborn State (year not } \\
\text { specified), Household } \\
\text { representative }\end{array}$} & & & $\begin{array}{l}\text { BF: } 19 \% \text { mothers } \\
\text { believe BF should } \\
\text { be discontinued }\end{array}$ & & & & \multirow{6}{*}{$\begin{array}{l}\text { Drugs: } 53 \% \text { of } \\
\text { mothers } \\
\text { reported } \\
\text { antibiotic and } \\
15 \% \text { reported } \\
\text { antidiarrheal } \\
\text { as treatment } \\
\text { for diarrhea }\end{array}$} \\
\hline & & & & & $\begin{array}{l}\text { Fluid: } 15 \% \text { believe } \\
\text { fluid should not be } \\
\text { offered during } \\
\text { episode }\end{array}$ & & & & \\
\hline & & & & & $\begin{array}{l}\text { Food: } 17 \% \text { believe } \\
\text { solid foods should } \\
\text { be withdrawn }\end{array}$ & & & & \\
\hline $\begin{array}{l}\text { Jinadu et al., } \\
1996 \text { [48] }\end{array}$ & Nigeria & $\begin{array}{l}\text { Mixed method study: } \\
\text { Structured interview } \\
\text { (335) and FGD (4), Rural } \\
\text { Yoruba communities of } \\
\text { Osuo State (year not } \\
\text { specified), Mothers of } \\
\text { children < } 5 \text { years }\end{array}$ & & & & $\begin{array}{l}\text { Fluid: More } \\
\text { mothers believe } \\
\text { fluids should not } \\
\text { be given for } \\
\text { watery diarrhea } \\
(65 \%) \text { compared } \\
\text { to bloody diarrhea } \\
(55 \%)\end{array}$ & & & \\
\hline \multirow[t]{2}{*}{$\begin{array}{l}\text { Ogunbiyi et al., } \\
2010 \text { [29] }\end{array}$} & \multirow[t]{2}{*}{ Nigeria } & \multirow{2}{*}{$\begin{array}{l}\text { Mixed method study: } \\
\text { Structured interviews } \\
(250) \text { and FGDs (2), } \\
\text { Ibadan 2003-2004, } \\
\text { Mothers of child } \\
<1 \text { year attending sick } \\
\text { baby/immunization } \\
\text { clinic of } 2 \text { health } \\
\text { facilities }\end{array}$} & \multirow[t]{2}{*}{$\begin{array}{l}\text { BF: "Cultural" reasons } \\
\text { for BF restriction - } \\
\text { passed from } \\
\text { generations }\end{array}$} & \multirow{2}{*}{$\begin{array}{l}\text { Food: Foods } \\
\text { withdrawn } \\
\text { because thought } \\
\text { to prolong the } \\
\text { duration of } \\
\text { diarrhea in the } \\
\text { child ( } 86 \% \text { ) and } \\
\text { induce vomiting/ } \\
\text { loss of appetite } \\
\text { (14\%) }\end{array}$} & $\begin{array}{l}\text { Food: Indigenous } \\
\text { foods rich in protein } \\
\text { withdrawn because } \\
\text { believed to } \\
\text { aggravate diarrhea }\end{array}$ & $\begin{array}{l}\text { BF: Overconsumption } \\
\text { of BM thought to } \\
\text { cause some diarrhea - } \\
\text { therefor reduce BF } \\
\text { frequency during } \\
\text { episode }\end{array}$ & & & \\
\hline & & & & & $\begin{array}{l}\text { Feeding: } 71 \% \\
\text { believe some food, } \\
\text { fluid, or breast milk } \\
\text { should be } \\
\text { withdrawn during } \\
\text { episode }\end{array}$ & $\begin{array}{l}\text { Food: Withdrawal } \\
\text { of other foods also } \\
\text { linked to mother's } \\
\text { perception of cause } \\
\text { of diarrhea }\end{array}$ & & & \\
\hline
\end{tabular}


Table 3 Beliefs, motivations, and context related to harmful practices by region and country (Continued)

\begin{tabular}{|c|c|c|c|c|c|c|c|}
\hline $\begin{array}{l}\text { Olakunle et al., } \\
2012 \text { [56] }\end{array}$ & Nigeria & $\begin{array}{l}\text { Quantitative study: } \\
\text { Structured interview } \\
\text { (186), llorin West Local } \\
\text { Government Area (year } \\
\text { not specified), Mothers } \\
\text { of children }<5 \text { years }\end{array}$ & $\begin{array}{l}\text { Feeding: Majority } \\
\text { said food restriction } \\
\text { was based on } \\
\text { personal view, but } \\
\text { some said received } \\
\text { information on food } \\
\text { restriction from } \\
\text { nurses }\end{array}$ & $\begin{array}{l}\text { Feeding: } 46 \% \text { of } \\
\text { mothers believe } \\
\text { "some food" should } \\
\text { be restricted during } \\
\text { episode }\end{array}$ & & & $\begin{array}{l}\text { Drug: } 17 \% \text { of } \\
\text { mothers } \\
\text { believe child } \\
\text { should be } \\
\text { treated with } \\
\text { antibiotic } \\
\text { during episode }\end{array}$ \\
\hline $\begin{array}{l}\text { Kauchali et al., } \\
2004 \text { [32] }\end{array}$ & $\begin{array}{l}\text { South } \\
\text { Africa }\end{array}$ & $\begin{array}{l}\text { Qualitative study: IDIs } \\
(16), \text { FGD (1), Case } \\
\text { histories (13) and card } \\
\text { sorting, Rural } \\
\text { Kwazulu-Natal 2001, } \\
\text { Caregivers of young } \\
\text { children, grandmothers, } \\
\text { CHWs }\end{array}$ & & & $\begin{array}{l}\text { BF: Perceived } \\
\text { causes of diarrhea } \\
\text { include "dirty" } \\
\text { breast milk requires } \\
\text { temporary stop in } \\
\text { breastfeeding }\end{array}$ & & \\
\hline \multirow[t]{2}{*}{$\begin{array}{l}\text { Friend du } \\
\text { Preeze et al., } \\
2013 \text { [72] }\end{array}$} & \multirow[t]{2}{*}{$\begin{array}{l}\text { South } \\
\text { Africa }\end{array}$} & \multirow{2}{*}{$\begin{array}{l}\text { Mixed method study: } \\
\text { IDIs (17), FGDs (5) and } \\
\text { structured interviews } \\
\text { (206), Johannesburg } \\
\text { and Soweto 2004, } \\
\text { Caregivers of children } \\
<6 \text { years in } \\
\text { longitudinal study and } \\
\text { health providers }\end{array}$} & & & & & $\begin{array}{l}\text { Drugs: Health } \\
\text { care workers } \\
\text { reported that } \\
\text { mothers } \\
\text { commonly use } \\
\text { non-prescribed } \\
\text { antibiotics }\end{array}$ \\
\hline & & & & & & & $\begin{array}{l}\text { Drugs: Demand } \\
\text { for modern } \\
\text { medicines is } \\
\text { high }\end{array}$ \\
\hline $\begin{array}{l}\text { Mwambete } \\
\text { et al., } 2010 \text { [118] }\end{array}$ & Tanzania & $\begin{array}{l}\text { Qualitative study: } \\
\text { Semi-structured } \\
\text { interviews (88), Dar es } \\
\text { Salaam 2007, } \\
\text { Mothers of children } \\
<5 \text { years }\end{array}$ & & & & $\begin{array}{l}35 \% \text { of } \\
\text { mothers } \\
\text { reported } \\
\text { metronidazole } \\
\text { as most } \\
\text { effective } \\
\text { chemotherapeutic } \\
\text { agent for } \\
\text { treating } \\
\text { diarrhea }\end{array}$ & $\begin{array}{l}\text { Drugs: } \\
\text { Metronidazole } \\
(43 \%) \text { and } \\
\text { Erythromycin + } \\
\text { Metronidazole } \\
(12 \%) \text { were } \\
\text { cited as } \\
\text { commonly } \\
\text { used } \\
\text { "therapeutic } \\
\text { agents" for } \\
\text { diarrhea } \\
\text { treatment }\end{array}$ \\
\hline
\end{tabular}


Table 3 Beliefs, motivations, and context related to harmful practices by region and country (Continued)

\begin{tabular}{|c|c|c|c|c|c|c|}
\hline \multicolumn{7}{|l|}{ South East Asia } \\
\hline \multirow[t]{2}{*}{$\begin{array}{l}\text { Mushtaque } \\
\text { et al., } 1991 \text { [55] }\end{array}$} & \multirow[t]{2}{*}{ Bangladesh } & \multirow{2}{*}{\multicolumn{2}{|c|}{$\begin{array}{l}\text { Qualitative study: } \\
\text { "Socioanthopologic } \\
\text { methods," Central } \\
\text { Bangladesh (year } \\
\text { not specified), villagers }\end{array}$}} & \multirow[t]{2}{*}{$\begin{array}{l}\text { Food: Certain types } \\
\text { of diarrhea require } \\
\text { withholding foods } \\
\text { that are normally } \\
\text { part of the diet }\end{array}$} & $\begin{array}{l}\text { General: Treatments } \\
\text { considered } \\
\text { appropriate depend } \\
\text { on the local } \\
\text { classification of the } \\
\text { diarrhea }\end{array}$ & \\
\hline & & & & & $\begin{array}{l}\text { BF: Injection of } \\
\text { breast milk into } \\
\text { woman used to } \\
\text { correct "polluted" } \\
\text { breast milk }\end{array}$ & \\
\hline \multirow[t]{2}{*}{$\begin{array}{l}\text { Singh et al., } \\
1994 \text { [43] }\end{array}$} & \multirow[t]{2}{*}{ India } & \multirow{2}{*}{\multicolumn{2}{|c|}{$\begin{array}{l}\text { Quantitative study: } \\
\text { Structured interviews } \\
\text { (208), Jaipur District } \\
\text { (year not specified), } \\
\text { Mothers of children } \\
<5 \text { years }\end{array}$}} & \multicolumn{3}{|l|}{$\begin{array}{l}\text { Feeding: Mothers } \\
\text { believe intestine } \\
\text { becomes weak and } \\
\text { child unable to } \\
\text { digest heavy foods } \\
\text { (roti and milk) } \\
\text { during episode }\end{array}$} \\
\hline & & & & \multicolumn{3}{|l|}{$\begin{array}{l}\text { Feeding: Tea water } \\
\text { and banana believed } \\
\text { to help reduce } \\
\text { frequency of } \\
\text { diarrhea }\end{array}$} \\
\hline $\begin{array}{l}\text { Chandrashekar } \\
\text { et al., } 1995 \text { [25] }\end{array}$ & India & $\begin{array}{l}\text { Qualitative study: } \\
\text { Semi-structured } \\
\text { interviews (300), Rural } \\
\text { South India 1991, } \\
\text { Mothers of children } \\
\text { age } 3 \text { days - } \\
17 \text { months }\end{array}$ & $\begin{array}{l}\text { Feeding: Elderly } \\
\text { relatives are source } \\
\text { of information on } \\
\text { feeding practices }\end{array}$ & & & $\begin{array}{l}\text { BF: Some } \\
\text { caregivers } \\
\text { believe } \\
\text { breastfeeding } \\
\text { should be } \\
\text { restricted } \\
\text { when mother } \\
\text { is experiencing } \\
\text { diarrhea or } \\
\text { respiratory } \\
\text { infection }\end{array}$ \\
\hline \multirow[t]{2}{*}{$\begin{array}{l}\text { Buch et al., } \\
1995 \text { [119] }\end{array}$} & \multirow[t]{2}{*}{ India } & \multirow{2}{*}{\multicolumn{2}{|c|}{$\begin{array}{l}\text { Quantitative study: } \\
\text { Structured interview } \\
\text { (1600), Kashmir 1992, } \\
\text { Caregivers of infants } \\
\text { with acute diarrhea } \\
\text { attending hospital } \\
\text { pediatric OPD }\end{array}$}} & $\begin{array}{l}\text { Feeding: } 19 \% \text { of } \\
\text { caregivers believe } \\
\text { child should have } \\
\text { complete dietary } \\
\text { restriction }\end{array}$ & & \multirow{2}{*}{$\begin{array}{l}\text { Drugs: } 55 \% \text { of } \\
\text { caregivers } \\
\text { believe } \\
\text { diarrhea } \\
\text { should be } \\
\text { treated with } \\
\text { antidiarrheal \& } \\
\text { antispasmodic } \\
\text { drugs, while } \\
32 \% \text { should } \\
\text { be treated } \\
\text { with drugs } \\
\text { and ORT }\end{array}$} \\
\hline & & & & $\begin{array}{l}\text { Fluid: } 77 \% \text { believe } \\
\text { milk should be } \\
\text { restricted }\end{array}$ & & \\
\hline
\end{tabular}

believe intestine

becomes weak a

child unable to

(roti and milk) 
Table 3 Beliefs, motivations, and context related to harmful practices by region and country (Continued)

\begin{tabular}{|c|c|c|c|c|c|c|}
\hline $\begin{array}{l}\text { Bhatia et al., } \\
1999 \text { [54] }\end{array}$ & India & $\begin{array}{l}\text { Quantitative study: } \\
\text { Structured interview } \\
\text { (120), Rural Chandigarh } \\
\text { 1996, Mothers of } \\
\text { children }<5 \text { years }\end{array}$ & & & $\begin{array}{l}\text { Feeding: } 47 \% \text { of } \\
\text { mothers believe } \\
\text { certain foods/fluids } \\
\text { should be restricted } \\
\text { including chapatti, } \\
\text { milk and pulses }\end{array}$ & \\
\hline $\begin{array}{l}\text { Datta et al., } \\
2001 \text { [120] }\end{array}$ & India & $\begin{array}{l}\text { Quantitative study: } \\
\text { Structured interview } \\
\text { (75), Rural Maharashtra } \\
\text { 2000, Caregivers of } \\
\text { children <5 years } \\
\text { attending hospital } \\
\text { pediatric OPD }\end{array}$ & & & $\begin{array}{l}\text { BF: } 16 \% \text { of } \\
\text { caregivers not aware } \\
\text { child has to be given } \\
\text { breastfeeding during } \\
\text { episode of diarrhea }\end{array}$ & \\
\hline \multirow[t]{2}{*}{$\begin{array}{l}\text { Vyas et al., } \\
2009 \text { [121] }\end{array}$} & \multirow[t]{2}{*}{ India } & \multirow{2}{*}{\multicolumn{3}{|c|}{$\begin{array}{l}\text { Quantitative study: } \\
\text { Structured interview } \\
\text { (380), Ganhinagar } \\
\text { district (year not } \\
\text { specified), Women of } \\
\text { reproductive age } \\
\text { (15-44) }\end{array}$}} & \multicolumn{2}{|l|}{$\begin{array}{l}\text { BF: } 52 \% \text { of women } \\
\text { did not know } \\
\text { breastfeeding should } \\
\text { be continued during } \\
\text { episode }\end{array}$} \\
\hline & & & & & \multicolumn{2}{|l|}{$\begin{array}{l}\text { Food: } 50 \% \text { did not } \\
\text { know other foods } \\
\text { should be continued }\end{array}$} \\
\hline $\begin{array}{l}\text { Bolam et al., } \\
1998 \text { [122] }\end{array}$ & Nepal & \multicolumn{3}{|l|}{$\begin{array}{l}\text { Quantitative study: } \\
\text { Structured interview } \\
\text { (105), Kathmandu } \\
\text { 1994-1996, Women } \\
\text { delivering at } \\
\text { Kathmandu General } \\
\text { Hospital }\end{array}$} & \multirow{2}{*}{\multicolumn{2}{|c|}{$\begin{array}{l}\text { BF: } 3 \text { months } \\
\text { postpartum, } 53 \% \\
\text { of mothers did not } \\
\text { know to continue } \\
\text { BF during episode }\end{array}$}} \\
\hline $\begin{array}{l}\text { Adhikari et al., } \\
2006 \text { [123] }\end{array}$ & Nepal & $\begin{array}{l}\text { Quantitative study: } \\
\text { Structured interview } \\
\text { (510), Kathmandu 2005, } \\
\text { Married women age } \\
\text { 18-38 from } 2 \text { village } \\
\text { development } \\
\text { committees }\end{array}$ & & $\begin{array}{l}\text { BF: } 7 \% \text { of } \\
\text { women believe } \\
\text { breastfeeding } \\
\text { aggravates } \\
\text { diarrhea }\end{array}$ & & \\
\hline \multirow[t]{2}{*}{$\begin{array}{l}\text { Ansari et al., } \\
2012 \text { [24] }\end{array}$} & \multirow[t]{2}{*}{ Nepal } & \multirow{2}{*}{$\begin{array}{l}\text { Qualitative study: FGDs } \\
\text { (2) and IDIs (8), Morang } \\
2010 \text {, Mothers of } \\
\text { children <45 months } \\
\text { with diarrhea in the } \\
\text { previous } 6 \text { months }\end{array}$} & \multirow{2}{*}{\multicolumn{2}{|c|}{$\begin{array}{l}\text { General: Elders } \\
\text { recommend } \\
\text { traditional } \\
\text { treatment practices }\end{array}$}} & $\begin{array}{l}\text { Food: Spicy, oily } \\
\text { and rotten food } \\
\text { commonly believed } \\
\text { to be harmful }\end{array}$ & \multirow{2}{*}{$\begin{array}{l}\text { General: Certain } \\
\text { types of diarrhea } \\
\text { are perceived to } \\
\text { be manageable } \\
\text { with ORS/SSW, } \\
\text { while others } \\
\text { require traditional/ } \\
\text { spiritual methods. }\end{array}$} \\
\hline & & & & & $\begin{array}{l}\text { BF: Breast milk } \\
\text { sometimes } \\
\text { considered harmful }\end{array}$ & \\
\hline
\end{tabular}

Food: $50 \%$ did no

ould be continued

of mothers did not

delivering at

Quantitative study:

Structured interview

Married women age

8-38 from 2 village 
Table 3 Beliefs, motivations, and context related to harmful practices by region and country (Continued)

\begin{tabular}{|c|c|c|c|c|c|c|c|}
\hline $\begin{array}{l}\text { Baclig et al., } \\
1990 \text { [58] }\end{array}$ & Thailand & $\begin{array}{l}\text { Mixed method study: } \\
\text { FGDs (2) and structured } \\
\text { interviews (98), Tambon } \\
\text { Korat and Koongyang } \\
\text { (year not specified), } \\
\text { Mothers and } \\
\text { grandmothers of } \\
\text { children < }<5 \text { years }\end{array}$ & & & $\begin{array}{l}\text { Feeding: Mothers } \\
\text { believe no } \\
\text { changes should } \\
\text { be made to the } \\
\text { child's diet to } \\
\text { manage poh } \\
\text { (a mild self-limiting } \\
\text { diarrhea) }\end{array}$ & & \\
\hline \multirow[t]{2}{*}{$\begin{array}{l}\text { Pylypa et al., } \\
2009 \text { [18] }\end{array}$} & \multirow[t]{2}{*}{ Thailand } & \multirow[t]{2}{*}{$\begin{array}{l}\text { Qualitative study: } \\
\text { Semi-structured } \\
\text { interviews (200) as part } \\
\text { of ethnographic study, } \\
\text { Rural Northeast } \\
\text { Thailand 2000-2001, } \\
\text { Caregivers of children } \\
<5 \text { years, traditional } \\
\text { healers, and health } \\
\text { providers }\end{array}$} & \multirow[t]{2}{*}{$\begin{array}{l}\text { General: } \\
\text { Grandmothers } \\
\text { and elders are } \\
\text { important sources } \\
\text { of information for } \\
\text { classifying/ } \\
\text { managing diarrhea }\end{array}$} & \multirow[t]{2}{*}{$\begin{array}{l}\text { Fluid/BF: Some } \\
\text { mothers restricted } \\
\text { water or breast } \\
\text { milk out of } \\
\text { concern that it } \\
\text { would make } \\
\text { diarrhea worse, } \\
\text { belief child could } \\
\text { not drink much } \\
\text { because he was } \\
\text { small, or would } \\
\text { vomit }\end{array}$} & $\begin{array}{l}\text { Food: Most } \\
\text { mothers didn't } \\
\text { change quantity/ } \\
\text { type of food } \\
\text { given for diarrhea } \\
\text { occurring in normal } \\
\text { developmental } \\
\text { stages (not illness) } \\
\text { although expected } \\
\text { children would eat } \\
\text { less in than normal }\end{array}$ & & \multirow[t]{2}{*}{$\begin{array}{l}\text { Medicines were } \\
\text { frequently } \\
\text { obtained from } \\
\text { health workers } \\
\text { most clinicians } \\
\text { consulted gave } \\
\text { antibiotics } \\
\text { routinely for } \\
\text { watery diarrhea } \\
\text { and for diarrhea } \\
\text { with fever }\end{array}$} \\
\hline & & & & & $\begin{array}{l}\text { Drugs: Medicines } \\
\text { were commonly } \\
\text { administered for } \\
\text { childhood diarrhea } \\
\text { considered illness }\end{array}$ & & \\
\hline \multicolumn{8}{|l|}{ Western Pacific } \\
\hline $\begin{array}{l}\text { Okumura et al., } \\
2002 \text { [70] }\end{array}$ & Vietnam & $\begin{array}{l}\text { Quantitative study: } \\
\text { Structured interviews } \\
\text { (505), } 4 \text { Provinces of } \\
\text { Vietnam 1997, } \\
\text { Mothers of children } \\
<5 \text { years }\end{array}$ & & & & & $\begin{array}{l}\text { Antibiotics to } \\
\text { be stocked at } \\
\text { home ( } 55 \% \text { of } \\
\text { households) } \\
\text { for various } \\
\text { anticipated } \\
\text { symptoms as } \\
\text { if they were } \\
\text { panaceas }\end{array}$ \\
\hline $\begin{array}{l}\text { Le et al., } \\
2011 \text { [69] }\end{array}$ & Vietnam & $\begin{array}{l}\text { Qualitative study: IDls } \\
\text { (5) and FGDs (4), Ha } \\
\text { Tay province (year not } \\
\text { specified), Mothers } \\
\text { of children < }<5 \text { years } \\
\text { and health workers/ } \\
\text { drug sellers }\end{array}$ & $\begin{array}{l}\text { Drugs: Drugs bought } \\
\text { on drug seller } \\
\text { recommendation or } \\
\text { previous prescriptions }\end{array}$ & & & $\begin{array}{l}\text { Western } \\
\text { medicine } \\
\text { considered } \\
\text { necessary but } \\
\text { more dangerous } \\
\text { than traditional } \\
\text { therapy }\end{array}$ & $\begin{array}{l}\text { Drugs are } \\
\text { available } \\
\text { without } \\
\text { prescription } \\
\text { and small } \\
\text { amount can } \\
\text { be purchased } \\
\text { to give for } \\
2-3 \text { days }\end{array}$ \\
\hline
\end{tabular}


Table 3 Beliefs, motivations, and context related to harmful practices by region and country (Continued)

\begin{tabular}{|c|c|c|c|c|c|c|}
\hline \multirow[t]{2}{*}{$\begin{array}{l}\text { Rheinlander } \\
\text { et al., } 2011 \text { [67] }\end{array}$} & \multirow[t]{2}{*}{ Vietnam } & \multirow{2}{*}{$\begin{array}{l}\text { Qualitative study: } \\
\text { Semi-structured } \\
\text { interviews (43), FGDs } \\
\text { (3), and observations, } \\
\text { Ethnic minorities in } \\
\text { Lao Cai 2008, } \\
\text { Caregivers of children } \\
<7 \text { years with diarrhea } \\
\text { in the past month }\end{array}$} & \multirow[t]{2}{*}{$\begin{array}{l}\text { General: Elders are in } \\
\text { charge of deciding, } \\
\text { preparing, and } \\
\text { administering } \\
\text { treatment for a } \\
\text { sick child }\end{array}$} & \multirow[t]{2}{*}{$\begin{array}{l}\text { Drugs: Medicines } \\
\text { chosen based on } \\
\text { perceived } \\
\text { compatibility with } \\
\text { the child and the } \\
\text { disease }\end{array}$} & \multirow[t]{2}{*}{$\begin{array}{l}\text { Antibiotics } \\
\text { perceived as } \\
\text { very powerful } \\
\text { and potentially } \\
\text { harmful } \\
\text { compared to } \\
\text { natural medicines }\end{array}$} & $\begin{array}{l}\text { Drugs: } \\
\text { common to } \\
\text { receive 2-4 } \\
\text { prescribed } \\
\text { drugs for } \\
\text { diarrhea }\end{array}$ \\
\hline & & & & & & $\begin{array}{l}\text { Drugs: To limit } \\
\text { intake and } \\
\text { harm of } \\
\text { western drugs, } \\
\text { caregivers } \\
\text { gave smaller } \\
\text { doses than } \\
\text { prescribed, or } \\
\text { shifted from } \\
\text { one drug to } \\
\text { another if } \\
\text { recovery was } \\
\text { slow }\end{array}$ \\
\hline
\end{tabular}

Beliefs, motivations, and context related to:

BF: Breastfeeding

Fluid: Fluid restriction

Feeding: Fluid, breastfeeding, and food restriction, or non-specific as to type of feeding

Drug: Use of modern medicines

General: Decision making around treatment or perception of diarrhea not specific to one of the harmful practice 
carbohydrate and high protein foods $[29,37,38,43$, 47-54]. Alternatively, in two studies some caregivers reported that specific foods were customary and should be given during a diarrhea episode to strengthen the bowel or soothe the stomach $[36,52]$. Some caregivers reported that restriction of certain foods was based on long held folk tradition [29, 47]. Others reported that diet alteration is based on the type or perceived cause of the diarrhea $[18,29,55]$. Elderly relatives, neighbors, and health care providers were reported to influence mothers' feeding practices in many contexts $[22,23,25$, $27,29,36,53,56,57]$. Some caregivers reported that a child's diet was not restricted during diarrhea because it was already limited [27, 44, 58]. One study reported mothers coaxed their child to eat more [36], but others reported some mothers of children with decreased appetite were unfamiliar with encouraging children to eat $[22,44]$ or had little time to prepare additional food because they were caring for the child [22]. One study suggested caregivers felt continued feeding was less important if they had been given some treatment at a health facility [31].

\section{Inappropriate medication use}

Many studies reported the use of drugs to treat diarrhea in children under five (Table 1, Column 7). The most commonly reported measures were the use of an antibiotic or antimicrobial, followed by use of any medicine, and the use of an antidiarrheal or antimotility agent. While antibiotics are recommended for treatment of dysentery or cholera, most studies did not differentiate between simple and dysenteric diarrhea when reporting on antibiotic use. The Lives Saved Tool (LiST) attributes $7 \%$ of diarrhea cases in children under 5 to dysentery [59], therefor it may be inferred that high antibiotic use rates are inclusive of inappropriate antibiotic use. A hospital-based study in Enugu, Nigeria highlights the difficultly of collecting information on the type of medicine used to treat diarrhea. The study reported that $70 \%$ of mothers misclassified antibiotics and analgesics as antimotility agents when self-reporting drugs used in diarrhea treatment [60]. Multiple studies outside of this review have shown that the accuracy of drug recall varies by questionnaire design and method of assessment [61].

Reported use of antidiarrheal and antimotility agents was generally lower than reported use of antibiotics. Use of antibiotics at any point in an episode ranged from 10$77 \%$. Antidiarrheal use ranged from 3-45\% of diarrhea episodes, with the exception of very high reported use (74 \%) in Egypt in 2002 [62]. Use of any drug for a diarrhea episode occurring in the previous 2 weeks ranged from $26-76 \%$. Studies that used a shorter reference period limited to the previous $24 \mathrm{~h}$ reported lower rates of drug use at around $20 \%$.
Multiple studies addressed variance in inappropriate medication use by factors including characteristics of the diarrhea episode, child, caregiver, and household (Table 2). A hospital-based study in Nigeria found children who had received an antibacterial or antidiarrheal at home presented to the hospital with more severe dehydration than those children who did not receive these drugs [60]. Antibiotic and/or antidiarrheal use were associated with seeking care outside of the home $[11,41]$ and use of ORT [60,63]. Two studies in Enugu, Nigeria reported conflicting associations between maternal education and antibiotic use [60, 64].

Caregivers reported using antibiotics and other drugs to treat diarrhea because they were accessible and believed to be efficacious (Table 3). Multiple studies reported caregiver beliefs that modern medicines are powerful [64-67], and more effective in treating diarrhea than ORS $[65,68]$. Multiple studies reported drugs were widely available and affordable in the public and private sector, typically without prescription [35, 38, 40, 44, 49, 52, 64, 69]. In many contexts, caregivers stocked drugs at home, purchasing them in advance or saving leftover medication from previous illnesses [33, 37, 38, 52, 70]. Caregivers perceived drugs to be cheaper and more accessible than ORS, particularly given the flexibility to purchase a few tablets for little money [64, 65, 71]. Use of antibiotics in the treatment of pediatric diarrhea has become routine for both health care providers and caregivers in some contexts $[18,40,66]$. Caregivers may have also influenced provider behavior as caregivers' preference for drug therapies creates pressure on providers to give medications in addition or instead of ORS [28, 33, 65, 72]. Drugs were given in sub-clinical doses in multiple studies $[67,69,73]$. It was common in studies for children to receive multiple drugs for a single episode of diarrhea, often from the same source [67, 74-77]. A study in Brazil found drugs were used more commonly to treat episodes of longer duration [63], although initial treatment of diarrhea at home with drugs was common in a study in Mali [78]. Multiple studies suggested treatment with modern medicines may be related to the perceived cause or type of diarrhea [18, 52, 60, 79-81]. Treatment seeking was often related to inappropriate use of medicine for diarrhea management $[33,57,62,82]$.

\section{Discussion}

This is the first review, to our knowledge, that addresses harmful practices related to fluids, feeding and medication use during episodes of childhood diarrhea. The findings indicate that there have been many studies both quantitative and qualitative - that have documented these harmful practices. However, reported prevalence varies greatly across study populations, and we were unable to identify clearly defined patterns 
across regions, countries, or time periods. A limited number of studies looked at the variation of these harmful practices across potential influencing factors, including characteristics of the diarrhea episode and child, caregiver, or household-level traits. Findings of association differed across studies.

The motivation for harmful practices during diarrhea treatment also appears to vary across populations, although studies consistently report general caregiver concern for their child's health and caregiver action to treat the illness to the best of their knowledge and abilities. Caregivers reported that their actions were based on the advice of health care providers, community members, or elderly relatives, as well as their own observations or understanding of the efficacy of certain treatments for diarrhea. Others reported following traditionally held beliefs on the causes and cures for specific diarrheal diseases.

Across studies, the measurement of harmful practices was inconsistent and not guided by a conceptual or theoretical framework. Most studies were focused on general practices in diarrhea treatment, and harmful practices were rarely a primary outcome of interest. This has limited the availability and quality of data on the topic. Variations in study design, sample populations, diarrhea episode reference periods, and measurement definitions make drawing comparisons and conclusions across studies challenging. This is further compounded by inconsistent quality in data collection and reporting. Most studies relied on sub-national population samples and many were limited to small sample sizes. The variation in treatment practices by perceived type of diarrhea highlights the importance of using local terminology in order to capture all episodes of diarrhea as perceived by the community [83]. Although the majority of studies included in this review used a recall period of diarrhea in the past two weeks, there was some variation ranging from the past $24 \mathrm{~h}$ to past six months or the "most recent" episode of diarrhea. Fischer-Walker and her colleagues highlight the importance of using a shorter recall period for capturing episodes of diarrhea of varying severity [83].

Although this systematic review highlighted limitations of existing research, the available evidence suggests that harmful practices in diarrhea treatment are common in certain populations. A multicountry analysis using MICS data from 28 countries between 2005-2007 reported the majority of mothers did not maintain their child's nutritional intake during illness [5]. Analysis of DHS data from 14 countries between 1986-2003 suggests a decreasing trend in continued feeding in a majority of countries [6]. These practices can reduce correct management of diarrheal disease in children and result in treatment failure and sustained nutritional deficits. The lack of consistency in sampling, measurement, and reporting identified in this literature review highlights the need to document harmful practices using standard methods of measurement and reporting. Going forward, studies in this area would benefit from the development and use of a broader conceptual framework to ensure that the research is theory-driven and regularly synthesized. Multi-country analyses using MICS and DHS data have been conducted in the past, but they have tended to focus on positive treatment practices rather than harmful practices [5, 6]. Assessing harmful practices with nationally representative data and standardized measurements, through the analysis of the most recently available DHS and MICS data, can contribute to the discussion on improved care of diarrheal disease in children under five.

The strengths of this literature review include applying a systematic process for searching and summarizing the literature, and accessing articles during a time frame in which global efforts focused on improving coverage. This review was limited by the inclusion of only peerreviewed literature and the exclusion of non-English language publications. Additionally, the quality of individual articles was not assessed, allowing for the potential inclusion of studies with misrepresentative findings.

\section{Conclusions}

Harmful practices in the management of childhood diarrhea are prevalent to varying degrees across cultures and include fluid and breastfeeding curtailment, food restriction, and inappropriate medication use. Inappropriate management of diarrhea episodes can result in higher risk of mortality through increased levels of dehydration or lasting health consequences as a result of nutritional restrictions or prolonged diarrhea illness. These practices must therefore be addressed as a matter of urgency in maternal, newborn and child health programs. These programs need to target not only the behaviors of child caregivers, but the broader social network, because our findings show that these practices are often informed by traditional beliefs, popular knowledge, and the instruction of authority figures, including elderly community members and health workers. Broader health systems interventions are also needed to address the alarming findings of high rates of inappropriate use of medications during diarrhea episodes. In addition, the global health community must do a better job or measuring the prevalence of these practices in standard ways, to produce evidence that can be used as the basis for action.

\section{Additional file}

Additional file 1: PubMed Search Terms. (PDF 68 kb)

Competing interests

The authors declare that they have no competing interests. 


\section{Authors' contributions}

$\mathrm{JB}$ and $\mathrm{HN}$ conceptualized the systematic review. EC developed the search criteria, conducted the systematic review, and prepared the first draft of the manuscript. JB, HN, and JP reviewed the search criteria and drafts of the manuscript. All authors approved the final version of the manuscript.

\section{Acknowledgements}

The authors would like to thank Christa Fischer-Walker and Cesar Victora for their helpful inputs on earlier drafts of this paper, and Peggy Gross for her technical assistance in developing literature search criteria.

This work was funded through a sub-grant from the U.S. Fund for UNICEF under the Countdown to 2015 for Maternal, Newborn and Child Survival grant from the Bill \& Melinda Gates Foundation. The funders had no role in the conceptualization of the paper or in the material presented.

\section{Author details}

${ }^{1}$ Institute for International Programs, Johns Hopkins Bloomberg School of Public Health, 615 North Wolfe Street, Baltimore, MD 21205, USA. ²Division of Policy and Strategy, Data and Analytics Section UNICEF, UNICEF, 3 UN Plaza, New York, NY 10017, USA.

\section{Received: 17 July 2014 Accepted: 6 August 2015} Published online: 18 August 2015

\section{References}

1. STATISTICS BY AREA/Child Survival and Health: Diarrhoea [http://www.child info.org/diarrhoea.html]

2. World Health Organization. The treatment of diarrhoea: a manual for physicians and other senior health workers. Geneva: WHO; 2005. p. 1-50.

3. Wardlaw T, Salama P, Brocklehurst C, Chopra M, Mason E. Diarrhoea: why children are still dying and what can be done. The Lancet. 2010;375(9718):870-2.

4. Wilson SE, Morris SS, Gilbert SS, Mosites E, Hackleman R, Weum KL, et al. Scaling up access to oral rehydration solution for diarrhea: Learning from historical experience in low-and high-performing countries. J Glob Health 2013:3:1.

5. Arabi M, Frongillo EA, Avula R, Mangasaryan N. Infant and young child feeding in developing countries. Child Dev. 2012;83(1):32-45.

6. Forsberg BC, Petzold MG, Tomson G, Allebeck P. Diarrhoea case management in low- and middle-income countries-an unfinished agenda. Bull World Health Organ. 2007;85(1):42-8.

7. Othero DM, Orago AS, Groenewegen T, Kaseje DO, Otengah PA. Home management of diarrhea among underfives in a rural community in Kenya: household perceptions and practices. East Afr J Public Health. 2008;5(3):142-6.

8. Kaatano GM, Muro Al, Medard M. Caretaker's perceptions, attitudes and practices regarding childhood febrile illness and diarrhoeal diseases among riparian communities of Lake Victoria, Tanzania. Tanzan Health Res Bull. 2006:8(3):155-61.

9. Mediratta RP, Feleke A, Moulton LH, Yifru S, Sack RB. Risk factors and case management of acute diarrhoea in North Gondar Zone, Ethiopia. J Health Popul Nutr. 2010;28(3):253-63.

10. Babaniyi OA, Maciak BJ, Wambai Z. Management of diarrhoea at the household level: a population-based survey in Suleja, Nigeria. East Afr Med J. 1994;71(8):531-5.

11. Wilson SE, Ouedraogo CT, Prince L, Ouedraogo A, Hess SY, Rouamba N, et al. Caregiver recognition of childhood diarrhea, care seeking behaviors and home treatment practices in rural Burkina Faso: a cross-sectional survey. PLoS One. 2012;7(3):e33273.

12. Bani IA, Saeed AA, Othman AA. Diarrhoea and child feeding practices in Saudi Arabia. Public Health Nutr. 2002;5(6):727-31.

13. Quadri F, Nasrin D, Khan A, Bokhari T, Tikmani SS, Nisar MI, et al. Health care use patterns for diarrhea in children in low-income periurban communities of karachi, Pakistan. Am J Trop Med Hyg. 2013;89(Suppl1):49-55.

14. Das SK, Nasrin D, Ahmed S, Wu Y, Ferdous F, Farzana FD, et al. Health careseeking behavior for childhood diarrhea in mirzapur, Rural Bangladesh. Am J Trop Med Hyg. 2013;89(Supp|1):62-8.

15. Nhampossa T, Mandomando I, Acacio S, Nhalungo D, Sacoor C, Nhacolo A, et al. Health care utilization and attitudes survey in cases of moderate-to-severe diarrhea among children ages 0-59 months in the District of Manhica, southern Mozambique. Am J Trop Med Hyg. 2013:89(1 Suppl):41-8.

16. Berisha M, Hoxha-Gashi S, Gashi M, Ramadani N. Maternal practice on management of acute diarrhea among children under five years old in Kosova. Turk Silahl Kuvvetleri Koruyucu Hekimlik Bulteni. 2009;8(5):369-72.

17. Olango P, Aboud F. Determinants of mothers' treatment of diarrhea in rural Ethiopia. Soc Sci Med. 1990;31(11):1245-9.

18. Pylypa J. Elder authority and the situational diagnosis of diarrheal disease as normal infant development in northeast Thailand. Qual Health Res. 2009;19(7):965-75

19. Ikpatt NW, Young MU. Preliminary study on the attitude of people in two states of Nigeria on diarrhoeal disease and its management. East Afr Med J. 1992:69(4):219-22.

20. Moawed SA, Saeed AA. Knowledge and practices of mothers about infants diarrheal episodes. Saudi Med J. 2000;21(12):1147-51.

21. Bachrach LR, Gardner JM. Caregiver knowledge, attitudes, and practices regarding childhood diarrhea and dehydration in Kingston. Jamaica Rev Panam Salud Publica. 2002;12(1):37-44.

22. Dearden KA, Quan LN, Do M, Marsh DR, Schroeder DG, Pachon H, et al. What influences health behavior? Learning from caregivers of young children in Viet Nam. Food Nutr Bull. 2002;23(4 SUPP):119-29.

23. Rasania SK, Gulati N, Sahgal K. Maternal beliefs regarding diet during acute diarrhea. Indian Pediatr. 1993;30(5):670-2.

24. Ansari M, Ibrahim MI, Hassali MA, Shankar PR, Koirala A, Thapa NJ. Mothers' beliefs and barriers about childhood diarrhea and its management in Morang district, Nepal. BMC Res Notes. 2012;5:576.

25. Chandrashekar S, Chakladar BK, Rao RS. Infant feeding-knowledge and attitudes in a rural area of Karnataka. Indian J Pediatr. 1995:62(6):707-12.

26. Okunribido OO, Brieger WR, Omotade O०, Adeyemo AA. Cultural perceptions of diarrhea and illness management choices among yoruba mothers in oyo state, Nigeria. Int Q Community Health Educ. 1997;17(3):309-18.

27. Ali M, Atkinson D, Underwood P. Determinants of use rate of oral rehydration therapy for management of childhood diarrhoea in rural Bangladesh. J Health Popul Nutr. 2000;18(2):103-8.

28. Prohmmo A, Cook LA, Murdoch DR. Childhood diarrhoea in a district in northeast Thailand: incidence and treatment choices. Asia Pac J Public Health. 2006;18(2):26-32.

29. Ogunbiyi BO, Akinyele IO. Knowledge and belief of nursing mothers on nutritional management of acute diarrhoea in infants, Ibadan, Nigeria. (Special Issue: Diversity of research.). Afr J Food Agric Nutr Dev. 2010:10(3):2291-304.

30. Kaltenthaler EC, Drasar BS. Understanding of hygiene behaviour and diarrhoea in two villages in Botswana. J Diarrhoeal Dis Res. 1996;14(2):75-80.

31. Shah MS, Ahmad A, Khalique N, Afzal S, Ansari MA, Khan Z. Home-based management of acute diarrhoeal disease in an urban slum of Aligarh, India. J Infect Dev Ctries. 2012;6(2):137-42.

32. Kauchali S, Rollins N, Van den Broeck J. Local beliefs about childhood diarrhoea: importance for healthcare and research. J Trop Pediatr. 2004:50(2):82-9.

33. Vazquez ML, Mosquera M, Kroeger A. People's concepts on diarrhea and dehydration in Nicaragua: the difficulty of the intercultural dialogue. Revista Brasileira de Saude Materno Infantil. 2002;2(3):223-37.

34. Nkwi PN. Perceptions and treatment of diarrhoeal diseases in Cameroon. J Diarrhoeal Dis Res. 1994;12(1):35-41.

35. Munthali AC. Change and continuity in the management of diarrhoeal diseases in under-five children in rural Malawi. Malawi Med J. $2005 ; 16(2): 43-6$

36. Almroth $\mathrm{S}$, Mohale M, Latham MC. Grandma ahead of her time: traditional ways of diarrhoea management in Lesotho. J Diarrhoeal Dis Res. 1997;15(3):167-72

37. Azim SM, Rahaman MM. Home management of childhood diarrhoea in rural Afghanistan: a study in Urgun, Paktika Province. J Diarrhoeal Dis Res. 1993;11(3):161-4

38. Omotade OO, Adeyemo AA, Kayode CM, Oladepo O. Treatment of childhood diarrhoea in Nigeria: need for adaptation of health policy and programmes to cultural norms. J Health Popul Nutr. 2000;18(3):139-44.

39. Oyoo A, Burstrom B, Forsberg B, Makhulo J. Rapid feedback from household surveys in PHC planning: An example from Kenya. Health Policy Plan. 1991;6(4):380-3. 
40. Perez-Cuevas R, Guiscafre H, Romero G, Rodriguez L, Gutierrez G. Mothers' healthseeking behaviour in acute diarrhoea in Tlaxcala, Mexico. J Diarrhoeal Dis Res. 1996;14(4):260-8.

41. Omore R, O'Reilly CE, Williamson J, Moke F, Were V, Farag TH, et al. Health careseeking behavior during childhood diarrheal illness: results of health care utilization and attitudes surveys of caretakers in western Kenya, 2007-2010. Am J Trop Med Hyg. 2013;89(1 Suppl):29-40.

42. Olson CK, Blum LS, Patel KN, Oria PA, Feikin DR, Laserson KF, et al. Community case management of childhood diarrhea in a setting with declining use of oral rehydration therapy: findings from crosssectional studies among primary household caregivers, Kenya, 2007. Am J Trop Med Hyg. 2011;85(6):1134-40.

43. Singh MB. Maternal beliefs and practices regarding the diet and use of herbal medicines during measles and diarrhea in rural areas. Indian Pediatr. 1994;31(3):340-3.

44. Hudelson P, Aguilar E, Charaly MD, Marca D, Herrera M. Improving the home management of childhood diarrhoea in Bolivia. Int Q Community Health Educ. 1994;15(1):91-104.

45. Uchendu UO, Emodi IJ, Ikefuna AN. Pre-hospital management of diarrhoea among caregivers presenting at a tertiary health institution: implications for practice and health education. Afr Health Sci. 2011;11(1):41-7

46. Ahmed F, Farheen A, Ali I, Thakur M, Muzaffar A, Samina M. Management of diarrhea in under-fives at home and health facilities in Kashmir. Int J Health Sci (Qassim). 2009;3(2):171-5.

47. Ekanem EE, Akitoye CO. Child feeding by Nigerian mothers during acute diarrhoeal illness. J R Soc Health. 1990;110(5):164-5.

48. Jinadu MK, Odebiyi O, Fayewonyom BA. Feeding practices of mothers during childhood diarrhoea in a rural area of Nigeria. Trop Med Int Health. 1996;1(5):684-9.

49. McLennan JD. Home management of childhood diarrhoea in a poor periurban community in Dominican Republic. J Health Popul Nutr. 2002;20(3):245-54.

50. Ali NS, Azam SI, Noor R. Women's beliefs regarding food restrictions during common childhood illnesses: a hospital based study. J Ayub Med Coll Abbottabad. 2003:15(1):26-8.

51. Smith GD, Gorter A, Hoppenbrouwer J, Sweep A, Perez RM, Gonzalez $C$, et al. The cultural construction of childhood diarrhoea in rural Nicaragua: relevance for epidemiology and health promotion. Soc Sci Med. 1993;36(12):1613-24.

52. Martinez H, Saucedo G. Mothers' perceptions about childhood diarrhoea in rural Mexico. J Diarrhoeal Dis Res. 1991;9(3):235-43.

53. Amini-Ranjbar S, Bavafa B. Iranian mother's child feeding practices during diarrhea: A study in Kerman. Pakistan J Nutr. 2007;6(3):217-9.

54. Bhatia V, Swami HM, Bhatia M, Bhatia SP. Attitude and practices regarding diarrhoea in rural community in Chandigarh. Indian J Pediatr. 1999;66(4):499-503.

55. Mushtaque A, Chowdhury R, Kabir ZN. Folk terminology for diarrhea in rural Bangladesh. Rev Infect Dis. 1991;13(Suppl 4):S252-254.

56. Olakunle JM, Valentine UO, Kamaldeen AS, Buhari ASM. Assessment of mothers' knowledge of home management of childhood diarrhea in a Nigerian setting. Int J Pharmaceut Res Bio Sci. 2012;1(4):168-84

57. Langsten R, Hill K. Treatment of childhood diarrhea in rural Egypt. Soc Sci Med. 1995;40(7):989-1001.

58. Baclig PV, Patrick WK. The cultural definition of an infantile diarrhea in Tambon Korat and Koongyang, northeast Thailand: community perceptions in diarrhea control. Asia Pac J Public Health. 1990;4(1):59-64.

59. Walker CLF, Walker N. The Lives Saved Tool (LiST) as a model for diarrhea mortality reduction. BMC Med. 2014;12(1):70

60. Uchendu UO, Ikefuna AN, Emodi IJ. Medication use and abuse in childhood diarrhoeal diseases by caregivers reporting to a Nigerian tertiary health institution. South Afr J Child Health. 2009;3(3):83-9.

61. Gama H, Correia S, Lunet N. Questionnaire design and the recall of pharmacological treatments: a systematic review. Pharmacoepidemiol Drug Saf. 2009;18(3):175-87.

62. El-Gilany AH, Hammad S. Epidemiology of diarrhoeal diseases among children under age 5 years in Dakahlia, Egypt. East Mediterr Health $J$. 2005;11(4):762-75.

63. Strina A, Cairncross S, Prado MS, Teles CA, Barreto ML. Childhood diarrhoea symptoms, management and duration: observations from a longitudinal community study. Trans R Soc Trop Med Hyg. 2005;99(6):407-16.
64. Ekwochi U, Chinawa JM, Obi I, Obu HA, Agwu S. Use and/or misuse of antibiotics in management of diarrhea among children in Enugu, Southeast Nigeria. J Trop Pediatr. 2013;59(4):314-6.

65. Wongsaroj T, Thavornnunth J, Charanasri U. Study on the management of diarrhea in young children at community level in Thailand. J Med Assoc Thai. 1997;80(3):178-82

66. Hoa NQ, Ohman A, Lundborg CS, Chuc NTK. Drug use and health-seeking behavior for childhood illness in Vietnam-A qualitative study. Health Policy. 2007;82(3):320-9.

67. Rheinlander T, Samuelsen H, Dalsgaard A, Konradsen F. Perspectives on child diarrhoea management and health service use among ethnic minority caregivers in Vietnam. BMC Public Health. 2011;11:690.

68. Zwisler G, Simpson E, Moodley M. Treatment of diarrhea in young children: results from surveys on the perception and use of oral rehydration solutions, antibiotics, and other therapies in India and Kenya. J Glob Health. 2013;3(1):10403.

69. Le TH, Ottosson E, Nguyen TK, Kim BG, Allebeck P. Drug use and selfmedication among children with respiratory illness or diarrhea in a rural district in Vietnam: a qualitative study. J Multidiscip Healthc. 2011;4:329-36.

70. Okumura J, Wakai S, Umenai T. Drug utilisation and self-medication in rural communities in Vietnam. Soc Sci Med. 2002;54(12):1875-86.

71. Winch PJ, Gilroy KE, Doumbia S, Patterson AE, Daou Z, Diawara A, et al. Operational issues and trends associated with the pilot introduction of zinc for childhood diarrhoea in Bougouni district, Mali. J Health Popul Nutr. 2008;26(2):151-62.

72. Friend-du Preez N, Cameron N, Griffiths P. "So they believe that if the baby is sick you must give drugs..." The importance of medicines in healthseeking behaviour for childhood illnesses in urban South Africa. Soc Sci Med. 2013;92:43-52.

73. Baqui AH, Black RE, El Arifeen S, Yunus M, Zaman K, Begum N, et al. Zinc therapy for diarrhoea increased the use of oral rehydration therapy and reduced the use of antibiotics in Bangladeshi children. J Health Popul Nutr. 2004;22(4):440-2.

74. Okoro BA, Jones IO. Pattern of drug therapy in home management of diarrhoea in rural communities of Nigeria. J Diarrhoeal Dis Res. 1995;13(3):151-4

75. Jousilahti P, Madkour SM, Lambrechts T, Sherwin E. Diarrhoeal disease morbidity and home treatment practices in Egypt. Public Health. 1997;111(1):5-10

76. World Health Organization. Diarrhoeal diseases household case management survey, Nepal, June, 1990 (Extended WER). Geneva: WHO; 1991. p. 22.

77. Diarrhoeal disease control (CDD) and acute respiratory infections (ARI). Combined CDD/ARI/breast-feeding survey, 1992. Wkly Epidemiol Rec 1993;68(17):120-122

78. Ellis AA, Winch P, Daou Z, Gilroy KE, Swedberg E. Home management of childhood diarrhoea in southern Mali-implications for the introduction of zinc treatment. Soc Sci Med. 2007;64(3):701-12.

79. Gorter AC, Sanchez G, Pauw J, Perez RM, Sandiford P, Smith GD. Childhood diarrhoea in rural Nicaragua: beliefs and traditional health practices. Boletin de la Oficina Sanitaria Panamericana. 1995;119(5):377-90.

80. Hudelson PM. ORS and the treatment of childhood diarrhea in Managua, Nicaragua. Soc Sci Med. 1993;37(1):97-103.

81. Ene-Obong HN, Iroegbu CU, Uwaegbute AC. Perceived causes and management of diarrhoea in young children by market women in Enugu State, Nigeria. J Health Popul Nutr. 2000;18(2):97-102.

82. Alam MB, Ahmed FU, Rahman ME. Misuse of drugs in acute diarrhoea in under-five children. Bangladesh Med Res Counc Bull. 1998;24(2):27-31.

83. Fischer Walker $\mathrm{CL}$, Fontaine $\mathrm{O}$, Black RE. Measuring coverage in $\mathrm{MNCH}$ : current indicators for measuring coverage of diarrhea treatment interventions and opportunities for improvement. PLoS Med. 2013;10(5):e1001385.

84. Emond A, Pollock J, Da Costa N, Maranhao T, Macedo A. The effectiveness of community-based interventions to improve maternal and infant health in the Northeast of Brazil. Rev Panam Salud Publica. 2002;12(2):101-10.

85. Webb AL, Ramakrishnan U, Stein AD, Sellen DW, Merchant M, Martorell R. Greater years of maternal schooling and higher scores on academic achievement tests are independently associated with improved management of child diarrhea by rural Guatemalan mothers. Matern Child Health J. 2010;14(5):799-806. 
86. Martinez H, Ryan GW, Guiscafre H, Gutierrez G. An intercultural comparison of home case management of acute diarrhea in Mexico: implications for program planners. Arch Med Res. 1998;29(4):351-60.

87. Kristiansson C, Gotuzzo E, Rodriguez H, Bartoloni A, Strohmeyer M, Tomson $\mathrm{G}$, et al. Access to health care in relation to socioeconomic status in the Amazonian area of Peru. Int J Equity Health. 2009;8:11.

88. Langsten R, Hill K. Diarrhoeal disease, oral rehydration, and childhood mortality in rural Egypt. J Trop Pediatr. 1994;40(5):272-8.

89. Diarrhoeal Diseases Control Programme: diarrhoea morbidity and case management survey, Morocco. Weekly Epidemiological Record 1991, 66(13):89-91.

90. Morisky DE, Kar SB, Chaudhry AS, Chen KR, Shaheen M, Chickering K Update on ORS usage in Pakistan: results of a national study. Pakistan J Nutr. 2002;1(3):143-50

91. Nasrin D, Wu Y, Blackwelder WC, Farag TH, Saha D, Sow SO, et al. Health care seeking for childhood diarrhea in developing countries: evidence from seven sites in Africa and Asia. Am J Trop Med Hyg. 2013;89(1 Suppl):3-12.

92. Bella H, Ai-Freihi H, El-Mousan M, Danso KT, Sohaibani M, Khazindar MS. Knowledge, Attitudes and Practices related to Diarrhoea in Eastern Province, Saudi Arabia. J Family Community Med. 1994;1(1):40-4.

93. Al-Mazrou YY, Aziz KM, Khan MU, Farag MK, Al-Shehri SN. ORS use in diarrhoea in Saudi children: is it adequate? J Trop Pediatr. 1995;41(Suppl 1):53-8.

94. Ketsela T, Asfaw M, Belachew C. Knowledge and practice of mothers/caretakers towards diarrhoea and its treatment in rural communities in Ethiopia. Ethiop Med J. 1991;29(4):213-24.

95. Mash D, Aschenaki K, Kedamo T, Walternsperger K, Gebreyes K, Pasha O, et al. Community and facility surveys illuminate the pathway to child survival in Liben Woreda, Ethiopia. East Afr Med J. 2003;80(9):463-9.

96. Saha D, Akinsola A, Sharples K, Adeyemi MO, Antonio M, Imran S, et al. Health Care Utilization and Attitudes Survey: understanding diarrheal disease in rural Gambia. Am J Trop Med Hyg. 2013;89(1 Suppl):13-20.

97. Mirza NM, Caulfield LE, Black RE, Macharia WM. Risk factors for diarrheal duration. Am J Epidemiol. 1997:146(9):776-85.

98. Burton DC, Flannery B, Onyango B, Larson C, Alaii J, Zhang X, et al. Healthcare-seeking behaviour for common infectious disease-related illnesses in rural Kenya: a community-based house-to-house survey. J Health Popul Nutr. 2011;29(1):61-70.

99. Simpson E, Zwisler G, Moodley M. Survey of caregivers in Kenya to assess perceptions of zinc as a treatment for diarrhea in young children and adherence to recommended treatment behaviors. J Glob Health. 2013;3(1):10405.

100. Perez F, Ba H, Dastagire SG, Altmann M. The role of community health workers in improving child health programmes in Mali. BMC Int Health Hum Rights. 2009;9:28.

101. Edet EE. Fluid intake and feeding practices during diarrhoea in Odukpani, Nigeria. East Afr Med J. 1996;73(5):289-91.

102. Omokhodion FO, Oyemade A, Sridhar MK, Olaseha IO, Olawuyi JF. Diarrhoea in children of Nigerian market women: prevalence, knowledge of causes, and management. J Diarrhoeal Dis Res. 1998;16(3):194-200.

103. Ogunrinde OG, Raji T, Owolabi OA, Anigo KM. Knowledge, attitude and practice of home management of childhood diarrhoea among caregivers of under-5 children with diarrhoeal disease in Northwestern Nigeria. J Trop Pediatr. 2012;58(2):143-6.

104. Cooke ML, Nel ER, Cotton MF. Pre-hospital management and risk factors in children with acute diarrhoea admitted to a short-stay ward in an urban South African hospital with a high HIV burden. South Afr J Child Health. 2013;7(3):84-7.

105. Haroun HM, Mahfouz MS, El Mukhtar M, Salah A. Assessment of the effect of health education on mothers in Al Maki area, Gezira state, to improve homecare for children under five with diarrhea. J Family Community Med. 2010;17(3):141-6.

106. Taha AZ. Assessment of mother's knowledge and practice in use of oral rehydration solution for diarrhea in rural Bangladesh. Saudi Med J. 2002;23(8):904-8

107. Larson CP, Saha UR, Nazrul H. Impact monitoring of the national scale up of zinc treatment for childhood diarrhea in Bangladesh: repeat ecologic surveys. PLoS Med. 2009;6(11):e1000175.

108. Sood AK, Kapil U. Knowledge and practices among rural mothers in Haryana about childhood diarrhea. Indian J Pediatr. 1990;57(4):563-6.
109. Gupta N, Jain SK, Chawla U, Hossain S, Venkatesh S. An evaluation of diarrheal diseases and acute respiratory infections control programmes in a Delhi slum. Indian J Pediatr. 2007;74(5):471-6.

110. Diarrhoeal disease control programme. Household survey of diarrhoea case management, Nepal. Wkly Epidemiol Rec. 1991;66(37):273-6.

111. Jha N, Singh R, Baral D. Knowledge, attitude and practices of mothers regarding home management of acute diarrhoea in Sunsari, Nepal. Nepal Med Coll J. 2006:8(1):27-30

112. Hoan LT, Chuc NTK, Ottosson E, Allebeck P. Drug use among children under 5 with respiratory illness and/or diarrhoea in a rural district of Vietnam. Pharmacoepidemiol Drug Saf. 2009;18(6):448-53.

113. Larrea-Killinger C, Munoz A. The child's body without fluid: mother's knowledge and practices about hydration and rehydration in Salvador, Bahia, Brazil. J Epidemiol Community Health. 2013;67(6):498-507.

114. Granich R, Cantwell MF, Long K, Maldonado Y, Parsonnet J. Patterns of health seeking behavior during episodes of childhood diarrhea: a study of Tzotzil-speaking Mayans in the highlands of Chiapas, Mexico. Soc Sci Med. 1999;48(4):489-95.

115. Ecker $L$, Ochoa TJ, Vargas M, Del Valle $L J$, Ruiz J. Factors affecting caregivers' use of antibiotics available without a prescription in Peru. Pediatrics. 2013:131(6):e1771-1779.

116. Agha A, White F, Younus M, Kadir MM, Alir S, Fatmi Z. Eight key household practices of integrated management of childhood illnesses (IMCI) amongst mothers of children aged 6 to 59 months in Gambat, Sindh, Pakistan. J Pak Med Assoc. 2007;57(6):288-93.

117. Rasheed P. Perception of diarrhoeal diseases among mothers and mothersto-be: implications for health education in Saudi Arabia. Soc Sci Med. 1993:36(3):373-7.

118. Mwambete KD, Joseph R. Knowledge and perception of mothers and caregivers on childhood diarrhoea and its management in Temeke municipality, Tanzania. Tanzan J Health Res. 2010;12(1):47-54.

119. Buch NA, Hassan M, Bhat IA. Parental awareness and practices in acute diarrhea. Indian Pediatr. 1995:32(1):76-9.

120. Datta V, John R, Singh VP, Chaturvedi P. Maternal knowledge, attitude and practices towards diarrhea and oral rehydration therapy in rural Maharashtra. Indian J Pediatr. 2001;68(11):1035-7.

121. Sheetal V. Impact of education on rural women about preparing ORS and SSS: a study of the primary health centre, Uvarsad, Gandhinagar. Health Popul Perspect Issues. 2009;32(3):124-30.

122. Bolam A, Manandhar DS, Shrestha P, Ellis M, Costello AM. The effects of postnatal health education for mothers on infant care and family planning practices in Nepal: a randomised controlled trial. BMJ. 1998:316(7134):805-11.

123. Adhikari P, Dhungel S, Shrestha R, Khanal S. Knowledge attitude and practice (KAP) study regarding facts for life. Nepal Med Coll J. 2006;8(2):93-6.

\section{Submit your next manuscript to BioMed Central and take full advantage of:}

- Convenient online submission

- Thorough peer review

- No space constraints or color figure charges

- Immediate publication on acceptance

- Inclusion in PubMed, CAS, Scopus and Google Scholar

- Research which is freely available for redistribution 